\title{
Desintegration und Interpretation: Weil-V2-Sätze an der Schnittstelle zwischen Syntax, Semantik und Pragmatik
}

\author{
MAILIN ANTOMO \& MARKUS STEINBACH
}

Abstract

In spoken varieties of German, causal adverbial clauses introduced by weil are frequently used with V2 order. Interestingly, weil-V2 clauses are not simply a colloquial variant of standard verb-final clauses, but have also many specific syntactic, semantic, and pragmatic properties. On the one hand, weil-V2 clauses differ systematically from their verb-final counterparts. While V2 clauses yield causal interpretations not available for the corresponding verb-final clauses, verb-final clauses show a higher degree of syntactic integration and syntactic flexibility than V2 clauses. On the other hand, weil-V2 clauses share crucial formal and functional properties with other kinds of embedded V2 clauses such as V2-complement clauses and V2-relative clauses. Weil-V2 clauses can therefore be subsumed under the notion of 'embedded root phenomena'. In this paper, we develop an analysis that derives all syntactic, semantic, and pragmatic properties of weil-V2 clauses at the interfaces between syntax, semantics, and pragmatics from their paratactic syntactic structure and the assertional force potential triggered by $V$-to-C movement. The results of a questionnaire study provide independent empirical evidence for our analysis.

Keywords: Causal Adverbial Clauses, Verb-Second, Assertion, Coordination, (Dis)Integration, Pragmatic Blocking

\section{Einleitung}

In der gesprochenen Sprache können mit weil eingeleitete Sätze statt der für Nebensätze kanonischen Verbletztstellung (vgl. 1b) auch die für Matrixkontexte typische V2-Stellung aufweisen (vgl. 1a).

* Für hilfreiche Kommentare und Unterstützung bedanken wir uns herzlich bei Hardarik Blühdorn, Elena Castroviejo Miró, Hans-Martin Gärtner, Liliane Haegeman, Klaus von Heusinger, Anke Holler, Shin-Sook Kim, Jörg Meibauer, Ingo Reich, Matthias 
a. Ich sag so was nicht, weil man darf das nicht sagen.

b. Ich sag so was nicht, weil man das nicht sagen darf.

Weil-V2-Sätze (im Folgenden WV2) wie in (1a) unterscheiden sich in Bezug auf ihre Syntax, Semantik und Pragmatik systematisch von den entsprechenden integrierten weil-VL-Sätzen (im Folgenden integrierte WVL). Gleichzeitig teilen sich WV2 wesentliche formale und funktionale Eigenschaften mit anderen V2-Nebensätzen wie dem V2-Relativsatz (RV2) in (2a) oder dem V2-Komplementsatz (KV2) in (2b).

a. Ich kenne einen Linguisten, der kann nicht lesen.

b. Hans glaubt, Peter hat geraucht.

Bei WV2 handelt es sich also um kein isoliertes Phänomen. WV2 stehen im Spannungsfeld zwischen eingebetteten V2-Sätzen und integrierten WVL. Die Gemeinsamkeiten und Unterschiede zu WVL sind in der Literatur gut dokumentiert. Was in den meisten Analysen von WV2 allerdings nicht berücksichtigt wird, ist ein systematischer Vergleich mit anderen eingebetteten V2-Sätzen (vgl. Gaumann 1983, Günthner 1993, Wegener 1993 und Uhmann 1998; zwei Ausnahmen sind Antomo 2007, Holler 2008 und Breindl 2009). ${ }^{1}$

In diesem Aufsatz werden wir dagegen zeigen, dass sich die spezifischen syntaktischen, semantischen und pragmatischen Eigenschaften von WV2 kompositional an den Schnittstellen zwischen Syntax, Semantik und Pragmatik ableiten lassen, wenn man WV2 im Kontext anderer V2-Nebensätze analysiert. Dabei sind zwei Eigenschaften von WV2 zentral: Zum einen diskutieren wir die Frage, weshalb WV2 nicht in jedem Kontext verwendet werden können, in dem die Verwendung von integrierten WVL möglich ist. Die Erklärung dieser Restriktion basiert auf neueren Analysen der Semantik von V2. Die Bewegung des finiten Verbs in die zweite Satzposition entfaltet ein assertives Illokutionspotential, das WV2 in präsupponierenden Kontexten generell ausschließt. Zum anderen erläutern wir, warum WV2 eine systematische Mehrdeutigkeit aufweisen, die so bei integrierten WVL nicht zu finden ist. Zentral für die Erklärung dieser Eigenschaft ist die WV2 zugrundeliegende paratakti-

Schlesewsky, zwei anonymen Gutachtern und den Teilnehmer/innen der AG1 der 31. Jahrestagung der Deutschen Gesellschaft für Sprachwissenschaft in Osnabrück.

1. Holler (2008) und Breindl (2009) diskutieren WV2 ebenfalls im Kontext von anderen V2-Nebensätzen und kommen zu ähnlichen Ergebnissen. Im Gegensatz zu diesen beiden Aufsätzen argumentieren wir in unserem Aufsatz für eine kompositionale Analyse, die die spezifischen Eigenschaften von WV2 aus dem Zusammenspiel von Syntax, Semantik und Pragmatik ableitet (vgl. auch Küper 1991, Gärtner 2001, Truckenbrodt 2006a und Steinbach 2007). 
sche Struktur, die in Verbindung mit dem assertiven Potential von WV2 sozusagen als Nebenprodukt zu einer semantischen und pragmatischen Flexibilität führt, die integrierte WVL so nicht aufweisen. Die Unterschiede zwischen WV2 und integrierten WVL werden damit ohne konstruktionsspezifische Annahmen systematisch aus der spezifischen Strukturbedeutung von V2-Sätzen und der syntaktischen und prosodischen Desintegration von WV2 abgeleitet.

Im nächsten Abschnitt werden wir zunächst ausführlich die spezifischen Eigenschaften von WV2 im Kontext von V2-Relativ- und V2Komplementsätzen diskutieren und dabei einen umfassenden Überblick über die relevanten Daten geben. Aus diesen Eigenschaften leiten wir in Abschnitt 3 eine parataktische syntaktische Analyse für WV2 ab. In Abschnitt 4 werden wir dann die verschiedenen Lesarten von WV2 und deren Zugänglichkeit untersuchen. Anschließend werden wir in Abschnitt 5 zeigen, dass sich die spezifischen Beschränkungen von WV2 aus der mit der V2-Stellung verbundenen Semantik ergeben. In einem zweiten Schritt werden in Abschnitt 6 die besonderen Lesarten von WV2 aus ihrer syntaktischen und prosodischen Desintegration abgeleitet. In Abschnitt 7 stellen wir eine kleine Fragebogenstudie vor, mit der wir wesentliche Aspekte der Analyse überprüfen. Der letzte Abschnitt fasst die Ergebnisse der Arbeit kurz zusammen. ${ }^{2}$

\section{Eigenschaften von V2- und VL-Nebensätzen}

WV2 unterscheiden sich in vielerlei Hinsicht von integrierten WVL (vgl. Gaumann 1983, Günthner 1993, Wegener 1993, Pasch 1997 und Uhmann 1998). Interessanterweise sind viele dieser Unterschiede nicht spezifisch für weil-Sätze, sondern auch bei anderen Arten von finiten Nebensätzen mit variabler Verbstellung zu finden. Die Eigenschaften von KV2 werden ausführlich in Reis (1995, 1997), Frank (2000), Meinunger (2004) und Truckenbrodt (2006a) diskutiert. Eine umfassende Diskussion von RV2 findet sich in Gärtner $(2001,2002){ }^{3}$

2. Da die Schwerpunkte dieses Artikels auf dem Vergleich von WV2 mit anderen eingebetteten V2-Sätzen, auf der Untersuchung der Syntax, Semantik und Pragmatik von WV2 und auf der Ableitung der spezifischen Lesarten von WV2 aus ihrer syntaktischen Struktur liegen, werden wir in diesem Artikel weder lexikalische (da, denn) und funktionale Alternativen zu weil-Sätzen noch sprachhistorische und typologische Aspekte diskutieren. Prosodische Aspekte von WV2 werden an unterschiedlichen Stellen miteinbezogen.

3. Alternation zwischen VL- und V2-Stellung in abhängigen Sätzen ist zudem auch in Komplementsätzen von Präferenzprädikaten, in Attributsätzen von Nomen und in Sätzen, die von Konnektoren wie obwohl, wobei und während eingeleitet werden, möglich (vgl. Reis 1997, Günthner 1999, Frank 2000, Wegener 2000 und Pasch et al. 2003). Außerdem unterliegen V2-Sätze mit integrierten V1-Parenthesen denselben Beschränkungen wie V2-Nebensätze (vgl. Reis 1995 und Steinbach 2007). 
Viele der im Folgenden diskutierten Eigenschaften wurden in den oben erwähnten Beiträgen bereits für die einzelnen V2-Nebensatztypen dokumentiert, allerdings meist ohne einen systematischen Zusammenhang zwischen WV2 und dem Verhalten anderer V2-Nebensätze herzustellen. In diesem Abschnitt soll deshalb einführend ein systematischer Überblick über die Eigenschaften von WV2 im Kontext von RV2 und KV2 geliefert werden (vgl. auch Holler 2008). Dabei wird sich zeigen, dass sich im Deutschen Nebensätze mit V2-Stellung konsequent von integrierten Nebensätzen mit kanonischer VL-Stellung unterscheiden. Gleichzeitig teilen sich WV2, RV2 und KV2 wesentliche Eigenschaften. Im Folgenden werden wir syntaktisch ungrammatische Beispiele wie gewohnt mit einem,${ }^{*}$ bewerten und für semantisch-pragmatische Abweichungen ein „\# verwenden, wohl wissend, dass jede Bewertung auch immer von theoretischen Annahmen abhängt.

Beginnen wir mit der Positionierung der Nebensätze. WV2 treten ausschließlich nachgestellt auf, wohingegen integrierte WVL auch im Vorfeld stehen können (vgl. Uhmann 1998: 102).
a. Peter kommt zu spät, weil er (hat) keinen Parkplatz gefun- den (hat).
b. Weil er (*hat) keinen Parkplatz gefunden (hat), kommt Pe- ter zu spät.

Dass es sich bei der in (3) illustrierten Stellungsrestriktion um kein isoliertes Phänomen von WV2 handelt, zeigt Beispiel (4). Interessanterweise treten auch RV2 ausschließlich extraponiert auf (vgl. Gärtner 2001: 472).

a. Ich suche jemanden, den (nennen) sie Wolf-Jürgen (nennen).

b. Jemanden, den (*nennen) sie Wolf-Jürgen (nennen), suche ich.

Bei KV2 ist das Bild etwas komplexer: Beispiele wie (5a) mit einem vorangestellten KV2 sind grundsätzlich syntaktisch ambig, da es sich entweder um einen Matrixsatz mit vorangestelltem V2-Komplement oder aber um einen V2-Matrixsatz mit satzfinaler integrierter V1-Parenthese handeln könnte. Da beide Konstruktionen fast denselben Beschränkungen unterliegen und von denselben Verben lizenziert werden, gibt es nur wenige Kontexte wie beispielsweise enger Fokus auf dem Matrixsubjekt in $(5 b)$, in denen die parenthetische Lesart aus unabhängigen Gründen ausgeschlossen ist. Derartige Beispiele zeigen, dass auch KV2 ausschließlich nachgestellt auftreten (vgl. Brandt et al. 1992, Reis 1995, 1997, Frank 2000, Truckenbrodt 2006a und Steinbach 2007).
a. Peter geht nach Hause, glaubt Maria.
b. \#Peter geht nach Hause, glaubt MARIA 
Eingebettete V2-Sätze können zudem nicht durch Korrelate aus dem Mittelfeld gebunden werden (zu Korrelaten vgl. Pasch et al. 2003). Wird der V2-Nebensatz von einem Korrelat (i. e. deshalb und es) gebunden, so sind die Beispiele unter (6) nicht akzeptabel. RV2 unterliegen einer ähnlichen Beschränkung. Im Gegensatz zu einem VL-Relativsatz kann ein RV2 wie in (6c) nicht bei seiner Bezugskonstituente im Mittelfeld stehen.
a. Lisa kommt deshalb zu spät, weil sie (*hat) keinen Park- platz gefunden (hat).
b. Peter hat es geglaubt, \#Lisa hat wieder mit dem Rauchen gen hat. angefangen. / dass Lisa wieder mit dem Rauchen angefan-
c. Gestern hat Lisa einen Parkplatz, der (*war) noch frei (war), gefunden.

Eine weitere Gemeinsamkeit ist, dass die Koordination eines KV2, RV2 und WV2 mit einem entsprechenden VL-Nebensatz ausgeschlossen ist. Dies spricht dafür, dass V2-Nebensätze grundsätzlich einen anderen syntaktischen Status haben als ihre VL-Entsprechungen (für Beispiele von asymmetrischer Koordination im Deutschen vgl. Reich 2008).
a. *Hans ist auf der Party, [weil er die Gastgeberin kennt und weil er muss heute Abend nicht arbeiten].
b. *Maria glaubt, [dass Hans auf ihre Party kommt und Petra bringt ihren Mann mit].
c. *Hans sucht einen Gast, [der Ritchy heißt und der schuldet ihm 250 Euro].

Eingebettete V2-Sätze können außerdem nie im Skopus einer Negation oder eines inhärent negativen Prädikats wie in (8c) interpretiert werden. Aus diesem Grund ist Beispiel (8b) ambig, wohingegen der WV2 in Beispiel (8a) nur die enge Skopuslesart zulässt, welche besagt, dass Silke der Party fernbleibt. Da sowohl der KV2 in (8c) wie auch der RV2 in (8e) nicht dem Skopus der Matrixnegation entkommen können (vgl. Abschnitte 3 und 5), sind beide Sätze nicht wohlgeformt.
a. Silke geht nicht auf die Party, weil sie will ausspannen ... (*sondern weil sie will tanzen)
b. Silke geht nicht auf die Party, weil sie ausspannen will ... (sondern weil sie tanzen will)
c. \#Er hat nicht geglaubt/bezweifelt, Lisa hat wieder mit dem Rauchen angefangen.
d. Er hat nicht geglaubt/bezweifelt, dass Lisa wieder mit dem Rauchen angefangen hat.


e. \#Lisa schaut sich keine Sendungen an, in denen macht Dieter Bohlen mit.

f. Lisa schaut sich keine Sendungen an, in denen Dieter Bohlen mitmacht.

Eine weitere Skopusbeschränkung ist in (9) zu beobachten. Präsupponierende Fokuspartikeln wie nur können aus dem Matrixsatz keine Konstituente in einem V2-Nebensatz binden, sehr wohl aber im entsprechenden VL-Nebensatz (vgl. Gärtner 2001: 129).
a. Lisa war nur auf der Party, weil sie (*wollte) HANS sehen (wollte) ... (und nicht weil ...)
b. Peter hat nur geglaubt, \#LIsa hat mit dem Rauchen ange- fangen. / dass LIsa mit dem Rauchen angefangen hat.
c. Ich kenne nur Leute, die (\#lesen) CHOMskys Bücher (lesen).

Die Bindung einer Konstituente in einem V2-Nebensatz durch eine Fokuspartikel im Hauptsatz ist allerdings nicht generell ausgeschlossen. Im Gegensatz zu präsupponierenden Fokuspartikeln wie nur können assertierende Fokuspartikeln wie auch oder sogar eine Konstituente in KV2 wie in Beispiel (10b) und in RV2 wie in Beispiel (10c) binden. Interessanterweise können sie aber nicht in einen WV2 binden (vgl. 10a). Da nur WV2, wie wir weiter unten noch zeigen werden, syntaktisch und prosodisch desintegriert sind, ist hier c-Kommando aus dem Bezugssatz generell ausgeschlossen. Im Gegensatz dazu sind KV2 eindeutig in den Matrixsatz integriert, so dass c-Kommando möglich ist. Bei RV2 ist die Datenlage etwas komplexer. Auf der einen Seite ist c-Kommando in den V2-Relativsatz nicht generell möglich (vgl. Beispiel (19) weiter unten), auf der anderen Seite bilden RV2 im Unterschied zu WV2 zusammen mit ihrem Matrixsatz zumindest eine informationsstrukturelle Einheit, was zu einer Ausweitung der syntaktischen Domäne für c-Kommando führen kann (vgl. Gärtner 2001: 124, 2002). Wir kommen weiter unten noch einmal auf diesen Punkt zurück. ${ }^{4}$
a. *Lisa war auch auf der Party, weil sie wollte HANS sehen.
b. Peter hat sogar geglaubt, LIsa hat wieder mit dem Rauchen angefangen.
e. Ich kenne sogar Leute, die lesen CHOMskys Bücher.
4. Für RV2 gibt es eine weitere sehr ähnliche Beschränkung. Beispiel (i) zeigt, dass sie anders als VL-Relativsätze nur indefinite NPn modifizieren können. So dürfen sie weder im Skopus bestimmter Quantoren wie alle stehen, noch definite NPn wie die Sendungen modifizieren (vgl. Gärtner 2001: 114).
(i) a. \#Lisa schaut sich die/alle Sendungen an, in denen macht Dieter Bohlen mit.
b. Lisa schaut sich die/alle Sendungen an, in denen Dieter Bohlen mitmacht.


Diese Beispiele zeigen, dass V2-Nebensätze generell in präsupponierenden Kontexten ausgeschlossen sind. Deswegen können sie im Gegensatz zu VL-Nebensätzen auch nicht Teil des Hintergrunds sein (vgl. 11).

(11) a. A: Du gehst in die Oper, obwohl dein Exfreund zu Besuch kommt?

B: \#Ich gehe in die Oper, WEIL mein Exfreund kommt zu Besuch.

b. A: Peter glaubt doch nicht, dass Lisa wieder mit dem Rauchen angefangen hat?

B: \#Doch, Peter GLAUBT, Lisa hat wieder mit dem Rauchen angefangen.

c. A: Kennst du jemanden, der ein Fahrrad besitzt?

B. \#Ja, ich KENne jemanden, der besitzt ein Fahrrad.

Dass WV2 nicht Teil des Hintergrunds sein dürfen, zeigt sich auch in den beiden folgenden Beispielen. In dem Hörbeleg (12a) wird zweimal fast derselbe weil-Satz verwendet, so dass V2-Stellung im zweiten Satz blockiert ist. In (12b) ist die genannte Begründung Teil des gemeinsamen Hintergrundwissens, was ebenfalls zu einer Blockierung der V2-Stellung im Kausalsatz führt:

(12) a. Ich kaufe immer in Spanien ein, weil da ist alles viel billiger. Meine T-Shirts und so hab ich alle aus Spanien, weil's da billiger ist.

b. A: Kathrin hat morgen Geburtstag.

B: \#Ja, ich weiß. Ich muss gleich noch in die Stadt fahren und ein Geschenk kaufen, weil sie hat morgen Geburtstag.

Zudem können WV2 und RV2 nicht in Interrogativsätze integriert werden. Diese Beschränkung gilt nicht für KV2 (vgl. 13). Wir werden weiter unten dafür argumentieren, dass KV2 im Gegensatz zu WV2 und RV2 nicht von der Illokution des Matrixsatzes abhängen, da sie als internes Argument immer in Bezug auf das Matrixverb interpretiert werden. ${ }^{5}$

a. Bist du mir böse, weil ich (\#war) gestern nicht bei deinem Vortrag (war)?

5. Die Beschränkung in (13) gilt bei WV2 und RV2 auch für Ergänzungsfragen und Imperativsätze. WV2 verfügen allerdings über eine Lesart, und zwar die sprechaktbezogene Begründung, die nicht im Skopus der Illokution des Matrixsatzes steht und deshalb Interrogativsätze und Imperativsätze modifizieren kann. Auf diesen Punkt kommen wir in Abschnitt 4 und 5 zurück. 
b. Hat Peter geglaubt, Maria wird wieder mit dem Rauchen anfangen? / dass Maria wieder mit dem Rauchen anfangen wird?

c. Kennt Hans einen Mann, der (\#geht) jeden Abend ins Kino (geht)?

Eine weitere Eigenschaft von V2-Nebensätzen ist, dass sie nicht gut als Echo-Sätze verwendet werden können. Die Echo-Entscheidungsfragen in (14) zeigen, dass VL-Nebensätze in Echo-Kontexten besser sind als ihre V2-Entsprechungen. Da Echo-Sätze Äußerungen über Äußerungen sind, muss immer eine Originaläußerung rekonstruiert werden. Dieser Bezug zu einer gegebenen Originaläußerung schließt wieder V2-Stellung im eingebetteten Satz aus (vgl. Reis 1992, Altmann 1993, Iwata 2003 und Graf 2007).

(14) a. A: Ich bin beim Zahnarzt, weil man hat mir einen Zahn ausgeschlagen.

B: Weil man (\#hat) dir einen Zahn ausgeschlagen (hat)?

b. A: Peter glaubt, Annika ist in die Stadt gegangen.

B: \#Annika ist in die Stadt gegangen? Das glaubt er? Dass Annika in die Stadt gegangen ist? Das glaubt er?

c. A: Ich suche jemanden, den nennen sie Wolf-Jürgen.

B: (Du suchst) jemanden, den (\#nennen) sie Wolf-Jürgen (nennen)?

Ein weiterer Unterschied zwischen VL- und V2-Nebensätzen besteht darin, dass V2-Nebensätze in Frage-Antwort-Paaren als Bezugssatzellipsen weniger optimal zu sein scheinen als die entsprechenden VL-Nebensätze (vgl. Reis 1997).
a. A: Warum bist du denn zu spät gekommen?
B: Weil ich (\#habe) keinen Parkplatz gefunden (habe).
b. A: Und was hat Annika geglaubt?
B: \#Der Wasserschaden ist an allem Schuld. / Dass der Wasserschaden an allem Schuld ist.
c. A: Was liest denn Hans-Martin gerne?
B: Bücher, die (\#haben) mit Linguistik zu tun (haben).

Trotz aller bisher aufgeführten Gemeinsamkeiten unterscheiden sich die einzelnen Typen von V2-Nebensätzen aber auch untereinander. Auf der einen Seite kontrastieren KV2 syntaktisch mit WV2 und mit RV2, auf der anderen Seite unterscheiden sich WV2 von RV2 und KV2. Wenden 
wir uns zuerst kurz den Besonderheiten von WV2 zu. Wir haben schon in Beispiel (10) gesehen, dass nur bei WV2 Bindung durch eine assertierende Fokuspartikel ausgeschlossen ist. Ein weiterer Unterschied betrifft die prosodische Integration. Im Gegensatz zu WV2 sind RV2 und KV2 wie die entsprechenden VL-Sätze intonatorisch in ihren Matrixsatz integriert. Sie bilden mit diesem zusammen eine Fokus-Hintergrund-Gliederung (im Folgenden FHG) und werden ohne prosodische Pause angeschlossen (vgl. Gärtner 2001: 98 und Reis 1997: 123). Der Bezugssatz endet mit steigender Intonation $\left(, /^{\circ}\right)$ und signalisiert so, dass die informationsstrukturelle Einheit noch nicht abgeschlossen ist.
a. Felix sagte, (/) dass Lisa wieder raucht.
b. Felix sagte, (/) Lisa raucht wieder.
c. Das Blatt hat eine Seite, (/) die ganz schwarz ist.
d. Das Blatt hat eine Seite, (/) die ist ganz schwarz.

Dies bedeutet, dass Relativ- und Komplementsätze unabhängig von der Verbstellung mit ihrem Bezugssatz immer eine prosodische und informationsstrukturelle Einheit bilden. In weil-Sätzen liegen die Dinge etwas anders: Während WVL typischerweise intonatorisch in ihren Matrixsatz integriert und somit Teil einer größeren FHG sind (vgl. 17b), bilden WV2 normalerweise eine eigene intonatorische Einheit mit einem eigenen Satzakzent und einer von ihrem Bezugssatz unabhängigen FHG. Wie in Beispiel (17a) ist der nachgestellte V2-Satz dabei häufig durch eine Pause von seinem Matrixsatz getrennt, der mit fallender Intonation $\left(, l^{\circ}\right)$ endet. Somit werden zwei intonatorische Einheiten produziert (vgl. Günthner 1993: 43 und Wegener 1993: 294). In Abschnitt 4 werden wir zeigen, dass auch nachgestellte WVL intonatorisch desintegriert werden können.
a. Silke verkauft ihre alten Sachen auf dem Flohmarkt, (\) weil sie braucht Geld.
b. Silke verkauft ihre alten Sachen auf dem Flohmarkt, (/) weil sie Geld braucht.

Noch nicht eindeutig geklärt ist, ob nach dem Konnektor weil eine intonatorische Pause oder eine prosodische Längung des Konnektors obligatorisch sind. Zudem ist nicht ganz klar, ob diese Pause aus der V2-Stellung im Nebensatz resultiert oder nicht. Während u. a. Buscha (1989: 126), Küper (1993: 41) und Gärtner (2001: 107) die Intonationspause als obligatorischen Bestandteil der Konstruktion betrachten, beobachten Wegener (1993: 291), Pasch (1997: 255), Scheutz (1998: 102) und Breindl (2009), dass eine Pause nach weil zwar möglich, aber nicht unbedingt 
zwingend ist. So kann der WV2 in Beispiel (18a) ohne semantischen Unterschied mit oder ohne Intonationspause ,- ' nach dem Komplementierer realisiert werden. Dass aber zumindest eine intonatorische Grenze zwischen weil und der satzinitialen Position des V2-Satzes existiert, zeigt Beispiel (18b). Die Klitisierung eines schwachen enklitischen Pronomens wie ' $m$ an weil ist nur in integrierten WVL, nicht aber in WV2 möglich (vgl. Wegener 1993, zu klitischen Pronomen im Deutschen vgl. Gärtner \& Steinbach 2003). Im nächsten Abschnitt werden wir dafür argumentieren, dass WV2 eine parataktische syntaktische Struktur zugrunde liegt. In WV2 befindet sich zwischen dem Konnektor und dem klitischen Pronomen im Vorfeld des zweiten Satzes eine Satzgrenze (CP), die eine Klitisierung des Pronomens blockiert. In integrierten WVL steht weil dagegen in $\mathrm{C}^{\circ}$ des eingebetteten Satzes (der Wackernagelposition) und ist damit ein optimaler Träger für das enklitische Pronomen am linken Rand des Mittelfelds. Die Enklitisierung eines Pronomens an den Konnektor weil ist in WV2 folglich aus syntaktischen Gründen ausgeschlossen. ${ }^{6}$

a. Kommt er? Weil (-) er hat's versprochen.

b. Er freut sich, weil'm (??ham) seine Eltern n'Fahrrad g'schenkt (ham).

Unabhängig davon, ob die prosodische Pause obligatorisch ist oder nicht, lässt sich festhalten, dass WV2 anders als KV2 und RV2 prosodisch nicht integriert sein müssen. Dies bedeutet, dass KV2 syntaktisch und prosodisch integriert sind, RV2 zumindest prosodisch integriert sein müssen und WV2 weder syntaktisch noch prosodisch integriert sind. Der Zusammenhang zwischen Integration und Interpretation wird in Abschnitt 4 noch einmal aufgegriffen.

Kommen wir nun zu den Besonderheiten von KV2, die sie von WV2 und RV2 unterscheiden. In (13) haben wir bereits gezeigt, dass einzig KV2 in Interrogativsätze eingebettet werden können. Ein weiterer syntaktischer Unterschied betrifft die Bindungsverhältnisse. Während WV2 und RV2 keine Quantoren-Variablen-Bindung zulassen, ist dies bei KV2 genauso wie bei VL-Nebensätzen problemlos möglich. ${ }^{7}$

6. Für die syntaktische Desintegration spricht auch, dass WV2 anders als WVL zumindest in manchen Kontexten Topik-Drop zulassen (Sören Schalowski, p.c.).

(i) a. Ich bin ganz schön müde, weil (-) hab heute viel zu viel gearbeitet.

b. *Ich bin ganz schön müde, weil (-) heute viel zu viel gearbeitet hab.

7. Derselbe Kontrast findet sich auch bei Prinzip C-Bindungseffekten. Koreferenz zwischen einem eingebetteten Eigennamen und einem Pronomen im Matrixsatz ist in WV2 und in RV2 besser als in KV2 (vgl. auch Gärtner 2001: 104).

(i) a. In jenem Sommer verlernte sie ${ }_{1}$ zu lachen, weil Maria ${ }_{1}$ war schwer krank.

b. * Sie $_{1}$ glaubte, Annika ${ }_{1}$ würde nicht eingeladen werden.

c. In Köln traf er ${ }_{1}$ Leute, die haben Hans ${ }_{1}$ nicht erkannt. 
(19) a. Niemand ${ }_{1}$ war verärgert, weil er $_{1}$ (*wurde) nicht eingeladen (wurde).

b. Jeder ${ }_{1}$ denkt, er ${ }_{1}$ kommt zu kurz. / dass er ${ }_{1}$ zu kurz kommt.

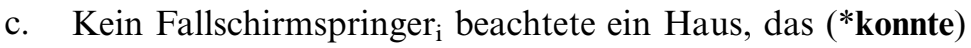
$\mathrm{er}_{\mathrm{i}}$ schlecht sehen (konnte).

In Komplementsätzen kann demnach unabhängig von der Verbstellung ein Quantor aus dem Matrixsatz eine Variable im eingebetteten Satz binden. KV2 unterscheiden sich bezüglich ihres Bindungsverhaltens folglich nicht von ihren VL-Gegenstücken (vgl. Meinunger 2004: 314). Bei Relativ- und Kausalsätzen blockiert dagegen die V2-Stellung die Bindungsrelation (vgl. Wegener 1993: 294, Uhmann 1998: 108 f. und Gärtner 2001: 104). KV2 sind also im Gegensatz zu den beiden anderen Typen von V2Nebensätzen eindeutig syntaktisch in den Matrixsatz integriert.

Die bisher diskutierten Daten haben gezeigt, dass sich eingebettete V2Sätze trotz einiger Unterschiede wesentliche syntaktische, semantische und pragmatische Eigenschaften teilen, so dass es nahe liegt, WV2 nicht als isoliertes Phänomen, sondern immer im Kontext anderer V2-Nebensätze zu untersuchen. Folgende Tabelle gibt eine Übersicht über die unterschiedlichen Eigenschaften der hier untersuchten Sätze.

Die Tabelle macht deutlich, dass sich V2-Nebensätze systematisch von VL-Nebensätzen unterscheiden. Daraus können wir schließen, dass V2und VL-Nebensätze unterschiedlich stark in die Matrixstruktur integriert

Tabelle 1: Eigenschaften von VL- und V2-Nebensätzen

\begin{tabular}{|l|c|c|c|c|}
\hline & VL-Nebensatz & KV2 & RV2 & WV2 \\
\hline Position im VF & + & - & - & - \\
\hline Korrelat im MF & + & - & - & - \\
\hline Koordination mit VL & + & - & - & - \\
\hline Matrixnegation & + & - & - & - \\
\hline Präsuppon. Fokuspartikel & + & - & - & - \\
\hline Hintergrund & + & - & - & - \\
\hline Echofragen & + & - & - & - \\
\hline Integration in Interrogativsatz & + & + & - & - \\
\hline Q/V-Bindung & + & + & - & - \\
\hline Assertierende Fokuspartikel & + & + & + & - \\
\hline Intonatorisch integriert & + & + & + & $+/-$ \\
\hline
\end{tabular}


sind. VL-Nebensätze sind grundsätzlich syntaktisch, semantisch und pragmatisch stärker in ihren Bezugssatz integriert als V2-Nebensätze. Darüber hinaus existieren aber auch einige Unterschiede zwischen den verschiedenen Arten von V2-Nebensätzen. Von den drei in diesem Abschnitt besprochenen V2-Nebensätzen sind WV2 am stärksten desintegriert. Hinzu kommt, dass sich die interne Oberflächenstruktur der einzelnen V2-Nebensätze unterschiedlich stark von den entsprechenden VL-Nebensätzen unterscheidet. Während sich bei einem RV2 lediglich das Verb in die zweite Position bewegt, wird bei einem KV2 zudem die subordinierende Konjunktion getilgt (complementizer drop). In WV2 wird die satzeinleitende Konjunktion nicht getilgt, sie ändert aber ihren syntaktischen Status von einer subordinierenden zu einer koordinierenden Konjunktion, so dass sich WV2 syntaktisch am stärksten von ihren VL-Gegenstücken unterscheiden. Die Syntax von V2-Nebensätzen ist Thema des nächsten Abschnitts.

\section{Die Syntax von V2-Nebensätzen}

Reis (1997) analysiert KV2 als VP-Adjunkte. Dies bedeutet, dass KV2 zwar als subordinierte Strukturen in den Matrixsatz integriert sind, so dass c-Kommando in den V2-Nebensatz möglich ist, sie sich aber gleichzeitig auch von kanonischen VL-Nebensätzen in VP-interner Komplementposition unterscheiden. Für RV2 schlägt Gärtner (2001) eine parataktische Analyse vor, die sich gut auf WV2 übertragen lässt. Während es bei RV2 auch Argumente gegen diese parataktische Analyse gibt (vgl. Gärtner 2002), ist die parataktische Struktur von WV2 relativ eindeutig (vgl. Höhle 1986, Uhmann 1998, Breindl 2009). So dürfen WV2 nicht im Skopus der Matrixnegation interpretiert werden (vgl. 8) und sie sind intonatorisch desintegriert (vgl. 17). Zudem ist in WV2, anders als in KV2 und RV2, die beide keine einleitende Konjunktion haben, eine satzeinleitende Konjunktion in Form des Konnektors weil vorhanden. Weil kann als Satzkoordinator analysiert werden, der syntaktisch zwei selbständige Matrixsätze verbindet (vgl. Abschnitt 5), so dass wir es mit einer eindeutig parataktischen Struktur zu tun haben. In Anlehnung an Gärtners (2001) parataktische Analyse von RV2 nehmen wir an, dass weil, wie in (20) dargestellt, die Kopfposition einer funktionalen Phrase $\pi \mathrm{P}$ mit dem V2-Adverbialsatz als Komplement und dem Bezugssatz als Spezifikator einnimmt. Im Unterschied zur parataktischen Analyse von RV2, wo $\pi_{\mathrm{REL}}^{\circ}$ phonologisch leer ist, ist $\pi^{\circ}$ in WV2 durch weil lexikalisch gefüllt. In (20a) verbindet weil damit zwei selbständige Hauptsätze. In Abschnitt 6 werden wir die Semantik dieser parataktischen Struktur genauer diskutieren (zum Status von $\pi^{0}$ vgl. Gärtner 2001). 

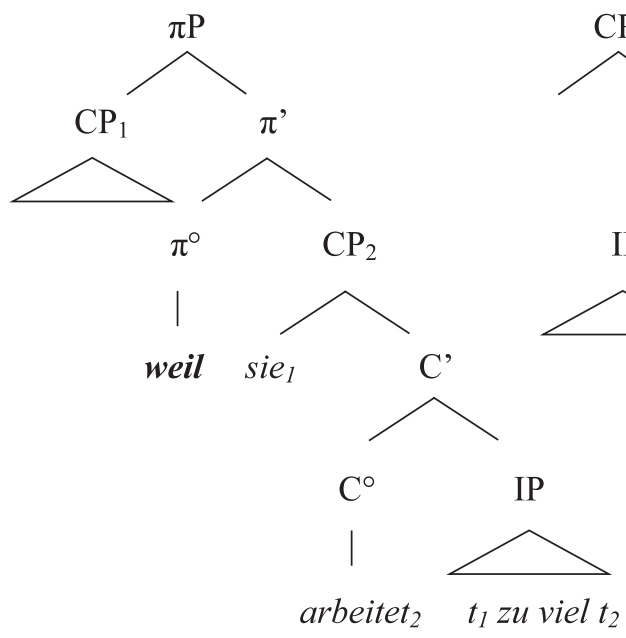
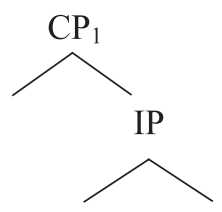

IP $\quad \mathrm{CP}_{2}$
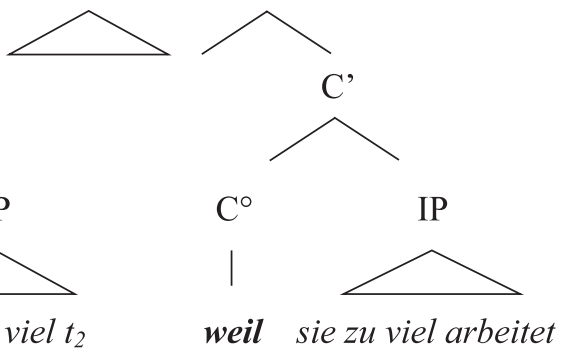

Es spricht außerdem einiges dafür anzunehmen, dass WV2 eine andere interne syntaktische Struktur haben als WVL. Wie in Beispiel (21) dargestellt, erlauben nur WV2, nicht aber WVL, Left Dislocation, was ein Hinweis dafür ist, dass WV2 eine reicher artikulierte CP besitzen als WVL (zur internen Struktur von Adverbialsätzen vgl. Haegeman 2003).

(21) Lass uns lieber Tim einladen, weil den Lukas, den (kann) ich gar nicht leiden (*kann).

Für unsere weitere Analyse ist die interne Struktur der V2-Sätze jedoch unerheblich, ebenso wenig ist die exakte Darstellung der parataktischen Struktur von WV2 relevant. Zentral ist dagegen die Asymmetrie zwischen syntaktisch integrierten VL-Nebensätzen und syntaktisch desintegrierten V2-Nebensätzen, unabhängig davon, in welcher Form diese implementiert ist. Mit WVL und WV2 liegen zwei völlig unterschiedliche Satzstrukturen vor. WVL sind syntaktisch integrierte Sätze mit einer subordinierenden Konjunktion und VL-Stellung. Im Gegensatz dazu sind WV2 syntaktisch desintegrierte Sätze mit V2-Stellung, die durch eine koordinierende Konjunktion mit dem Bezugssatz verbunden sind. Die syntaktischen Eigenschaften der drei V2-Nebensätze sind in Tabelle 2 noch einmal zusammengefasst. Dabei liegt in allen drei Fällen eine syntaktische Asymmetrie zwischen der VL- und der V2-Variante vor. 
Tabelle 2: Syntaktische Basispositionen von VL- und V2-Nebensätzen

\begin{tabular}{|l|l|l|}
\hline & VL-Nebensatz & V2-Nebensatz \\
\hline Komplementsatz & $\mathrm{V}^{\circ}$-Komplement & VP-Adjunktion \\
\hline Relativsatz & DP-Adjunkt & CP-Koordination \\
\hline Weil-Satz & VP-Adjunkt & CP-Koordination \\
\hline
\end{tabular}

Damit lassen sich zunächst die ersten fünf der in Tabelle 1 dargestellten Eigenschaften von WV2 ableiten. Da es sich bei den adverbialen WV2 um parataktische Strukturen handelt, in denen der WV2 immer das zweite Konjunkt ist (i. e. $\mathrm{CP}_{2}$ in Struktur (20a)), stehen WV2 obligatorisch satzfinal. Aufgrund der syntaktischen Desintegration erlauben sie zudem keine Korrelate und können nicht im Skopus der Matrixnegation und von Fokuspartikeln stehen. Die hier skizzierte syntaktische Analyse ist allerdings nur die halbe Geschichte. Sie erklärt weder die spezifischen Lesarten von WV2, noch die fast identischen Beschränkungen von KV2 und (je nach Analyse) von RV2. Zumindest KV2 sind, wie die Bindungsdaten zeigen, eindeutig in den Matrixsatz integriert, so dass sie syntaktisch im Skopus der Matrixnegation und von präsupponierenden Fokuspartikeln stehen. Wir werden in Abschnitt 5 dafür argumentieren, dass sich die in Tabelle 1 dargestellten Beschränkungen von V2-Nebensätzen nicht allein aus ihrer Syntax, sondern vor allem aus der Strukturbedeutung des eingebetteten V2-Satzes ergeben. Die syntaktische Desintegration, die bei WV2 besonders stark ausgeprägt ist, ist somit auch ein Reflex eines allgemeineren Phänomens von sogenannten abhängigen (oder eingebetteten) Hauptsätzen (vgl. Hooper \& Thompson 1973, Wechsler 1991, Reis 1997 und Gärtner 2002). Die unterschiedlichen Grade der Integration sind in Abbildung 1 für WV2, RV2 und KV2 vereinfacht zusammengefasst. KV2 und RV2 sind dabei einerseits syntaktisch, prosodisch und informationsstrukturell stärker in den Matrixsatz integriert als WV2, andererseits aber weniger stark integriert als die entsprechenden VL-Sätze.

schwach integriert

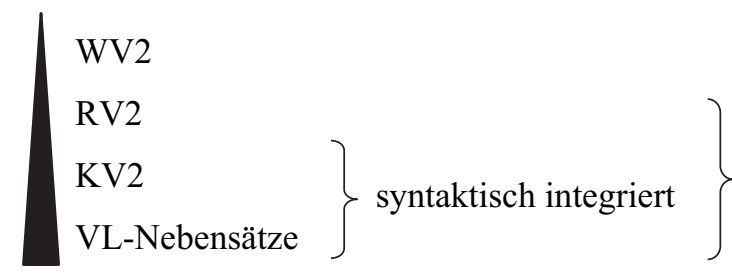

prosodisch und informationsstrukturell integriert

stark integriert

Abbildung 1: Grade der Integration von Nebensätzen 
Abbildung 1 zeigt, dass WV2 in jeder Hinsicht optimal desintegrierte Nebensätze sind. Integration ist ein komplexer Begriff, der mehrere Dimensionen hat. Finite Nebensätze können zum einen syntaktisch und/ oder prosodisch (des-)integriert sein. Zum anderen geht mit einer syntaktischen und prosodischen Desintegration normalerweise auch eine gewisse semantische Flexibilität und pragmatische Eigenständigkeit einher. Bevor wir auf die semantischen und pragmatischen Aspekte der Desintegration von WV2 in den nächsten beiden Abschnitten genauer eingehen, wollen wir die unterschiedlichen Grade der Integration finiter Sätze abschließend kurz an drei Beispielen illustrieren (infinite Nebensätze und Parenthesen berücksichtigen wir hier nicht):

a) VL-Komplementsätze sind optimal integrierte Strukturen, die prosodisch, syntaktisch, semantisch (als Argument des Matrixverbs) und pragmatisch (als Teil der FHG und hinsichtlich ihres assertiven Potentials, vgl. Abschnitt 5) ein Bestandteil des Matrixsatzes sind.

b) WV2 sind dagegen optimal desintegrierte Strukturen: Prosodisch endet der Bezugssatz mit einer fallenden Intonation (vgl. 17), syntaktisch liegt eine parataktische Struktur mit V2-Stellung vor, semantisch weisen WV2 eine Mehrdeutigkeit auf, die bei integrierten WVL nicht $\mathrm{zu}$ finden ist, und pragmatisch bilden WV2 eine eigene FHG und haben aufgrund der V2-Stellung auch ein eigenes assertives Potential.

c) RV2 sind stärker in den Matrixsatz integriert als WV2, aber weniger stark als VL-Komplementsätze: Einerseits sind RV2 wie VL-Komplementsätze prosodisch und semantisch integriert. Der Bezugssatz endet mit einer steigenden Intonation (vgl. 16) und der RV2 wird wie der entsprechende VL-Relativsatz als restriktiver Relativsatz interpretiert. Zudem sind RV2 Teil der FHG des Matrixsatzes. Andererseits sind RV2 wie WV2 syntaktisch und auch pragmatisch desintegriert, da eine parataktische Struktur mit V2-Stellung vorliegt und der RV2 deshalb ebenfalls ein eigenes assertives Potential besitzt.

\section{Die Interpretation von WV2}

Obwohl sich die drei Arten von V2-Nebensätzen zahlreiche Eigenschaften teilen, erhalten sie doch recht unterschiedliche Interpretationen. KV2 sind wie ihre VL-Entsprechungen direkte Objekte, die eine thematische Rolle vom Matrixprädikat erhalten. Da KV2 in Reis' (1997) Analyse syntaktisch Adjunkte sind, kann die thematische Rolle nicht wie bei VLKomplementen strukturell in der Komplementposition lizenziert werden, so dass der Nebensatz auf nichtkanonische Art lizenziert werden muss. Eine mögliche Analyse wäre, die Lizenzierungsbedingungen zu lockern, 
damit die nicht zugewiesene thematische Rolle in der Syntax an ein VPAdjunkt zugewiesen werden kann. Alternativ dazu könnte die thematische Rolle erst in der Semantik lizenziert werden. In diesem Fall hätte das Matrixverb eine syntaktisch nicht lizenzierte thematische Rolle und der V2-Nebensatz wäre ein normales Adjunkt, das in der Syntax keine thematische Rolle erhält (vgl. Reis 1997, Frank 2000 und Steinbach 2007).

Auch RV2 werden wie VL-Relativsätze als restriktive Relativsätze interpretiert (vgl. Gärtner 2001, 2002). Nimmt man wie Gärtner (2001) eine parataktische Analyse für RV2 an, dann muss der Relativsatz semantisch in den Bezugssatz rekonstruiert werden (für eine entsprechende Analyse vgl. Gärtner 2001).

Von bestimmten durch die V2-Stellung ausgelösten semantischen Effekten einmal abgesehen (vgl. Tabelle 1) unterscheiden sich KV2 und RV2 folglich nicht wesentlich von ihren VL-Entsprechungen. Dies ist bei weil-Sätzen anders. WV2 und integrierte WVL erhalten zwar beide eine kausale Lesart, trotzdem gibt es grundlegende semantische Unterschiede zwischen diesen beiden Typen von weil-Sätzen. Im Gegensatz zu integrierten WVL können WV2 unterschiedliche Aspekte einer Äußerung kausal modifizieren (zur kausalen Semantik von weil vgl. Ballweg 2004, Lohnstein 2004 und Blühdorn 2006). WV2 unterscheiden sich daher nicht nur syntaktisch, sondern auch semantisch und pragmatisch systematisch von integrierten WVL. WV2 weisen als optimal desintegrierte Nebensätze unmarkiert eine Ambiguität auf, die bei integrierten WVL nicht zu finden ist. So können WV2 neben der für integrierte WVL typischen propositionalen Lesart je nach Kontext auch eine epistemische oder sprechaktbezogene Lesart erhalten. Die Zugänglichkeit einer bestimmten Interpretation hängt wesentlich vom Grad der Integration des Nebensatzes in seinen Bezugssatz ab. Je enger die syntaktische Anbindung an den Matrixsatz ist, desto eingeschränkter ist die Zugänglichkeit bestimmter Lesarten und umgekehrt (zur Ambiguität von kausalen Konnektoren vgl. Sweetser 1990, Keller 1993, Breul 1997, Pasch 1997, Scheutz 1998, Pasch et al. 2003, Haegeman 2006, Breindl 2009 und Volodina 2009).

Im Folgenden diskutieren wir die drei unterschiedliche Interpretationen von weil-Sätzen - propositionale, epistemische und sprechaktbezogene Begründung - in Abhängigkeit von der (Des-)Integration des Nebensatzes. Dabei konzentrieren wir uns vor allem auf den Kontrast zwischen WV2 und integrierten WVL. Wir werden allerdings noch zeigen, dass WVL auch prosodisch desintegriert auftreten können. In diesem Fall weisen sie eine mit WV2 vergleichbare semantische Flexibilität auf.

Propositionale Begründung: Ein integrierter WVL liefert typischerweise eine Begründung für den in der vorhergehenden Proposition ausge- 
drückten Sachverhalt. In (22) behauptet die Sprecherin S, dass der Grund des Zurückfahrens am Mittag (q) der graue Himmel (p) war. Eine solche Begründung auf der Sachverhaltsebene wird als propositional bezeichnet. Auch WV2 können in einer propositionalen Begründungsrelation zu ihrem Bezugssatz stehen. So kann der satzfinale WVL in (22a) durch einen V2-Satz in (22b) ersetzt werden.

(22) a. Mittags sind wir zurückgefahren, weil der Himmel ganz grau war.

b. Mittags sind wir zurückgefahren, weil der Himmel war ganz grau.

c. q weil $p$

In Abschnitt 2 haben wir jedoch bereits gezeigt, dass ein WVL nicht in jedem Kontext durch einen WV2 substituiert werden kann. Da es sich dabei hauptsächlich um Beschränkungen handelt, die ebenfalls für KV2 und RV2 gültig sind, können diese nicht durch die spezifische Syntax von WV2 erklärt werden. Stattdessen ergeben sich diese Beschränkungen aus der Strukturbedeutung von eingebetteten V2-Sätzen, die wir im nächsten Abschnitt ausführlicher diskutieren werden.

Epistemische Einstellungsbegründung: So genannte epistemische weilSätze wie (23a) begründen nicht den in der vorhergehenden Proposition ausgedrückten Sachverhalt, sondern liefern Begründungen auf der illokutionären Ebene für die Sprechereinstellung. Mit dem Kausalsatz in (23a) teilt S mit, weshalb sie annimmt, dass es geschneit habe, indem sie die Wissensgrundlage schafft, die zu der im ersten Teilsatz dargelegten Inferenz führt. Da die Straße weiß ist, schließt sie darauf, dass es geschneit haben muss (vgl. Küper 1991, Günthner 1993, Wegener 1993, Pasch 1997, Scheutz 1998, Uhmann 1998: 116 ff. und Blühdorn 2008).
a. Es hat geschneit, weil die Straße ist ganz weiß.
b. \#Es hat geschneit, (/) weil die Straße ganz weiß ist.
c. \#Es hat deswegen geschneit, (/) weil die Straße ganz weiß ist.
d. \#Weil die Straße ganz weiß ist, (/) hat es geschneit.
e. Es hat geSCHNEIT, ( $($ ) weil die STRAße ganz weiß ist.
f. (S glaubt q) weil $p$

Uhmann (1998: 117) und Wegener (1993: 295) zufolge können epistemische Einstellungsbegründungen nur durch WV2 ausgedrückt werden. Das ist zutreffend, wenn man WV2 mit integrierten WVL kontrastiert. Ist ein WVL eindeutig integriert, so ist die epistemische Lesart nicht zugänglich und er kann nur propositional interpretiert werden, was aber in Sätzen wie (23b-d) aufgrund unseres Weltwissens zu semantischen Ab- 
weichungen führt. Neben der integrierenden Intonation (/) in (23b) sind die Bindung des Nebensatzes durch ein Korrelat im Mittelfeld in (23c), sowie die Topikalisierung des Nebensatzes in (23d) eindeutige Signale für eine Integration des Satzes.

Diese Sätze zeigen, dass bei eindeutiger Integration des weil-Satzes nur die propositionale Lesart zugänglich ist. Ein WVL kann aber, wie schon erwähnt, auch prosodisch desintegriert realisiert werden. Der intonatorisch desintegrierte WVL in (23e) bildet eine eigene Intonationseinheit mit einem eigenen Nuklearakzent. In diesem Fall kann er als epistemische Einstellungsbegründung auf die illokutionäre Ebene bezogen werden. Es besteht folglich ein weiterer interpretatorischer Kontrast zwischen intonatorisch integrierten und desintegrierten WVL. Die epistemische Lesart eines weil-Satzes ist offensichtlich nur zugänglich, wenn der Satz syntaktisch und/oder prosodisch desintegriert ist (vgl. Pasch 1997, Scheutz 1998, Blühdorn 2008, Breindl 2009). Auf WV2 trifft dies immer $\mathrm{zu}$, da eine parataktische Struktur vorliegt (vgl. 20). WVL dagegen, die gewöhnlich in die CP ihres Bezugssatzes integriert sind, können nur dann eine epistemische Einstellungsbegründung ausdrücken, wenn durch eine markierte Prosodie signalisiert wird, dass es sich um einen desintegrierten Gebrauch handelt. Da in diesem Aufsatz die Analyse von WV2 im Mittelpunkt steht, werden wir im Folgenden prosodisch desintegrierte WVL nicht weitere berücksichtigen (zu desintegrierten WVL vgl. Reis 2006).

Während WVL präferiert propositional interpretiert werden und die epistemische Lesart nur im Falle eines unintegrierten Gebrauchs und bei entsprechenden Kontextbedingungen zugänglich ist, sind WV2 aufgrund ihrer syntaktischen Struktur optimal desintegriert und damit semantisch und pragmatisch flexibler, so dass sie grundsätzlich erst einmal ambig sind.

Sprechaktbezogene Begründung: Wie epistemische Einstellungsbegründungen beziehen sich sprechaktbezogene Begründungen nicht auf die propositionale, sondern auf die illokutionäre Ebene. Während aber epistemische WV2 begründen, wie $\mathrm{S}$ zu der im Matrixsatz ausgedrückten Annahme kommt, wird mit sprechaktbezogenen WV2 wie in (24a-c) die Realisierung des mit dem ersten Satz ausgeführten Sprechakts begründet. Für diese Lesart ist charakteristisch, dass der Bezugssatz und der nachgestellte WV2 unterschiedliche illokutionäre Potentiale aufweisen können. ${ }^{8}$

8. Da sprechaktbezogene WV2 nicht dasselbe illokutionäre Potential wie ihr Bezugssatz haben müssen, ist zu erwarten, dass sie auch interrogativ und imperativ sein können. Die folgenden beiden Beispiele zeigen, dass dies möglich ist.

(i) a. Peter hatte gestern einen Unfall. Weil hast du gesehen, wie sein Auto aussieht?

b. Hier hast du 5 Euro. Weil geh doch mal zum Kiosk und hol mir Zigaretten! 
(24) a. Ich habe den Job gekriegt. Weil das interessiert dich doch am meisten.

b. Bist du nervös? Weil du rauchst schon deine dritte Zigarette.

c. Vertrauen Sie diesem Mann nicht! Weil ich kenne ihn.

d. Vertrauen Sie diesem Mann nicht (deshalb), (/) weil ich ihn kenne.

e Bist du nervös? (\) Weil du schon deine dritte Zigarette rauchst.

f. (S äußert q) weil p

Beispiel (24d) (nach Thim-Mabrey 1982: 216) zeigt, dass integrierte WVL nur eine Lesart als propositionale Begründung zulassen (vgl. Küper 1991: 137, Günthner 1993: 41, Wegener 1993: 295). Die sprechaktbezogene Lesart von WVL ist jedoch wieder möglich, wenn der WVL wie in (24e) intonatorisch desintegriert ist.

Abbildung 2 gibt einen zusammenfassenden Überblick über die in diesem Abschnitt diskutierten Interpretationen eingebetteter V2-Sätze im Vergleich zu der Interpretation von VL-Sätzen.

1. KV2 $\Rightarrow$ Argument des Verbs

2. RV2 $\Rightarrow$ Restriktiver Relativsatz

(= VL-Komplementsatz)

3. WV2 a. $\Rightarrow$ propositionale Begründung

$(=$ VL-Relativsatz $)$

b. $\Rightarrow$ epistemische Einstellungsbegründung ( $\neq$ integrierte WVL)

c. $\Rightarrow$ sprechaktbezogene Begründung $\quad(\neq$ integrierte WVL $)$

Abbildung 2: Interpretation von V2-Nebensätzen

\section{Die Semantik von V2-Nebensätzen}

Wir haben bisher gesehen, dass sich WV2 systematisch von integrierten WVL unterscheiden. So können sie einerseits nicht in jedem Kontext einen integrierten WVL ersetzen (Tabelle 1), haben andererseits aber eine größere Interpretationsvielfalt als integrierte WVL (Abbildung 2). Die Ableitung dieser Besonderheiten von WV2 erfolgt dementsprechend in zwei Schritten. In diesem Abschnitt werden wir zeigen, dass V2-Nebensätze ein assertives Potential haben. Dieses assertive Potential ermöglicht es uns, zusammen mit der in Abschnitt 3 skizzierten syntaktischen Analyse die in Tabelle 1 diskutierten Beschränkungen von V2-Nebensätzen zu erklären. In nächsten Abschnitt werden wir dann die drei unterschiedlichen Lesarten von WV2 aus der parataktischen Struktur ableiten.

Auf das assertive Potential von WV2 wurde in der Literatur schon des Öfteren hingewiesen (vgl. Gaumann 1983, Günthner 1993, Pasch 1997, Scheutz 1998, 2001 und Pasch et al. 2003). Allerdings wurde bisher mit der Ausnahme von Antomo (2007), Holler (2008) und Breindl (2009) 
kein systematischer Zusammenhang zu der V2-Stellung in anderen Nebensätzen und generell zur Semantik der V2-Stellung hergestellt. In Abschnitt 2 haben wir schon gezeigt, dass die Unterschiede zwischen WV2 und WVL kein isoliertes Phänomen sind. Im Deutschen gibt es unterschiedliche Arten von V2-Nebensätzen, die sich nicht nur die Wortstellung, sondern auch gewisse semantische und pragmatische Eigenschaften mit uneingebetteten V2-Hauptsätzen teilen. Die Bewegung des finiten Verbs nach $\mathrm{C}$ löst sowohl in uneingebetteten als auch in eingebetteten Strukturen eine bestimmte Interpretation aus, so dass im Deutschen V2Sätze eine spezifische Strukturbedeutung haben. Im Folgenden werden wir uns auf die Ansätze von Gärtner $(2001,2002)$ und Truckenbrodt (2006a, b) beziehen, da beide eine elaborierte Analyse von eingebetteten RV2 und KV2 im Deutschen geben. Wir diskutieren dabei vor allem die für die Analyse von WV2 relevanten Aspekte. ${ }^{9}$

Eines der zentralen Formmerkmale des deutschen Satzmodussystems ist die Verbstellung (vgl. Altmann 1993). Bei allen drei Grundtypen in (25) befindet sich das finite Verb in der C-Position. Damit einher geht jeweils, dass die Sprecherin S vom Adressaten A etwas möchte. Mit der Äußerung eines Imperativsatzes wie (25a) möchte $\mathrm{S}$ normalerweise, dass A etwas tut. Mit der Äußerung eines Deklarativ- oder Interrogativsatzes möchte $\mathrm{S}$ hingegen, dass das gemeinsame Hintergrundwissen (der common ground, im Folgenden CG) verändert wird. Mit einem Deklarativsatz wie in (25b) fügt $\mathbf{S}$ selbst eine neue Information zum CG hinzu und möchte, dass A diese akzeptiert. Im Gegensatz dazu möchte S mit der Äußerung eines Interrogativsatzes wie (25c), dass A den CG um eine bestimmte Information erweitert (vgl. Brandt et al. 1992, Lohnstein 2000, Stalnaker 2002 und Truckenbrodt 2006a, b).
a. Öffne das Fenster!
,S möchte von A, dass A das Fenster öffnet.'
b. Annika hat das Fenster geöffnet. ,S möchte von $A$, dass es CG ist, dass Annika das Fenster geöffnet hat.'
c. Hat Annika das Fenster geöffnet?
,S möchte von A, dass es $\mathrm{CG}$ ist, ob Annika das Fenster geöffnet hat.'

9. Entsprechende Beispiele eingebetteter Hauptsätze finden sich auch in anderen Sprachen. Allerdings nutzen nur wenige Sprachen unterschiedliche Verbstellungen zur Markierung eingebetteter Hauptsätze. Weitere Strategien sind complementizer drop, Modusalternationen und die Verwendung bestimmter Konnektoren (zu eingebetteten Hauptsätzen vgl. auch Hooper \& Thompson 1973, Wechsler 1991, Breul 1997, Reis 1997, Frank 2000, Meinunger 2004 und Heycock 2005). 
Truckenbrodt (2006a) argumentiert nun, dass grammatische Merkmale in $\mathrm{C}$ mit einer bestimmten Interpretation interagieren, die sich wie folgt umschreiben lässt: ,S möchte (von A) (dass es Teil des CGs ist) dass/ob $\mathrm{p}^{c}$. Diese Interpretation wird durch Kontextindizes in $\mathrm{C}$ vermittelt, die in uneingebetteten Sätzen wie folgt aussehen:

$$
<\text { Deont }_{\mathrm{S}}(, \mathrm{x})_{1}(,<\text { Epist }>)_{2}>
$$

Dabei entspricht $<$ Deont $_{\mathbf{s}}>$ dem Wunsch der Sprecherin (S möchte) und , ${ }^{6}$ der Person, die Kontrolle über die mit dem Satz ausgedrückte Proposition hat. $<$ Epist $>$ legt wiederum fest, dass es Teil des CGs ist, dass oder ob p. Dies bedeutet, dass $<$ Epist $>$ zu einer (normalerweise von A kontrollierten) Erweiterung des CG führt. Im Gegensatz zu $<$ Deont $_{\mathbf{s}}>$ sind die letzten beiden Indizes von syntaktischen Eigenschaften des C-Systems abhängig: < Epist $>$ ist nur dann aktiv, wenn sich in $\mathrm{C}$ das finite Verb und/oder ein [+WH]-Merkmal befindet. Genauso wird die Variable x nur dann mit A identifiziert, wenn $\mathrm{C}$ ein nach Person flektiertes finites Verb enthält (vgl. Truckenbrodt 2006a: 265).

In a context index $<$ Deont $_{\mathrm{S}}(, \mathrm{x})(,<$ Epist $>)>$ in $\mathrm{C}$

a. Epist is present iff (i) $\mathrm{C}$ contains a finite verb with indicative or Konjunktiv II or (ii) $\mathrm{C} / \mathrm{CP}$ is marked [ $+\mathrm{WH}$ ].

b. $\quad \mathrm{x}=\mathrm{A}(\mathrm{ddressee})$ iff $\mathrm{C}$ contains a finite verb with person inflection.

Mit dieser Definition lässt sich nun der Imperativ in (25a) wie in (28a) dargestellt beschreiben - Bedingung (27a) trifft hier nicht $\mathrm{zu}$, da das Verb in $\mathrm{C}$ imperativisch flektiert und $\mathrm{C}$ nicht $[+\mathrm{WH}]$ markiert ist. Die entsprechenden Deklarative und Interrogative sind in (28b) und (28c) angegeben. Relevant ist hierbei, dass in Deklarativen und Interrogativen der Kontextindex $<$ Epist $>$ eine Kontextveränderung erzwingt, die von A kontrolliert wird.

$$
\begin{aligned}
& \text { a. Imperativ: }<\text { Deont }_{\mathrm{S}}, \mathrm{A}>\quad, S \text { möchte von } A \text {, }
\end{aligned}
$$

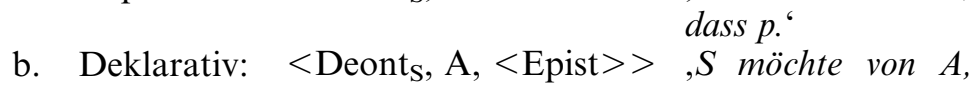

$$
\begin{aligned}
& \text { c. Interrogativ: }<\text { Deont }_{\mathrm{S}}, \mathrm{A},<\text { Epist }>>\text { ist, dass } p \text { möchte von } A \text {, }
\end{aligned}
$$

Dies bedeutet, dass der Bewegung des finiten Verbs (zusammen mit der WH-Markierung in C) im Deutschen eine zentrale Funktion bei der 
Satztypenmarkierung zukommt. Dies lässt sich gut an einem Vergleich von uneingebetteten VL- und V2-Sätzen illustrieren (vgl. Truckenbrodt 2006a). VL-Interrogative (deliberative Fragen) wie (29a) haben zwar aufgrund der Definition (27a) < Epist> aktiviert. Im Gegensatz zu V1-Interrogativen wie (25c) ist hier aber nicht die Variable x spezifiziert. Deshalb können sie nur in Kontexten verwendet werden, in denen S von A keine Antwort erwartet. Ein VL-Deklarativ wie (29b) unterscheidet sich noch stärker von seinem entsprechenden V2-Deklarativ in (25b). Da hier weder ein $[+\mathrm{WH}]-$ Merkmal noch das finite Verb in $\mathrm{C}$ sind, kann der Satz keine Veränderung des CG bewirken und es bleibt lediglich eine deontische (oder exklamative) Interpretation.

(29) a. [Kontext: S und A haben gemeinsam den Raum verlassen, nachdem sie Annika gebeten haben, das Fenster zu öffnen]. S: Ob Annika das Fenster aufgemacht hat?

b. Dass Annika (ja) das Fenster öffnet!

Wie lässt sich die Analyse uneingebetteter V2-Sätze nun auf eingebettete V2-Sätze übertragen? Nach Gärtner (2002) und Truckenbrodt (2006a) haben RV2 und KV2 genauso wie uneingebettete Sätze ein assertives Potential, lösen aber im Gegensatz zu letzteren kein eigenständiges Kontextupdate aus und können daher nicht als eigenständige Assertionen verwendet werden. Ihr assertives Potential (oder die proto-assertional force) wird im Matrixsatz absorbiert, so dass die V2-Nebensätze im Skopus der Matrixassertion (RV2) oder des assertiven Matrixverbs (KV2) interpretiert werden (zum Begriff der Absorption vgl. Gärtner 2002 und Truckenbrodt 2006a: 281 f.). In KV2 und RV2 checkt die V-nach-C-Bewegung demnach nur einen der drei Kontextindizes, und zwar $<$ Epist $>$. Die Indizes $<$ Deont $_{S}$, A $>$ sind hingegen auf Matrixkontexte beschränkt, da nur auf dieser Ebene $\mathrm{S}$ und A zur Verfügung stehen.

Bei WV2 sieht die Sache etwas anders aus. Wir haben bereits in Abschnitt 2 gezeigt, dass WV2 anders als RV2 und KV2 außerhalb der Matrixnegation interpretiert werden können (vgl. 8), außerdem bilden sie typischerweise eine eigene FHG (vgl. 16). Am eindeutigsten ist der Fall der sprechaktbezogenen WV2, die, wie in Beispiel (24) gezeigt, unterschiedliche Sprechakte wie Interrogativ- oder Imperativsätze modifizieren können. Zudem können sie wie in Beispiel (30) alleine, d. h. ohne Bezugssatz, verwendet werden, was ebenfalls auf ihren Status als eigenständige Assertionen hindeutet.

(30) Kontext: A gibt B ein Päckchen. A: Weil du hattest doch Geburtstag. 
Aber auch epistemische und propositionale WV2 bilden eigene assertive Sprechakte. So ist in Bezug auf die in Tabelle 1 dargestellten spezifischen Eigenschaften kein Unterschied zwischen den drei Verwendungen von WV2 zu erkennen, der auf unterschiedliche Einbettungsgrade schließen lassen könnte. ${ }^{10}$ Zwar können propositionale und epistemische WV2 nur assertive Bezugssätze modifizieren. Dies liegt aber darin begründet, dass sie einen assertierten Sachverhalt bzw. einen epistemischen Zustand als Argument benötigen, welche von Imperativ- oder Interrogativsätzen nicht geliefert werden. Und auch die Tatsache, dass WV2 kausale Modifikatoren sind, spricht nicht gegen eine Analyse als eigenständige Assertionen. Kausale Relationen zwischen zwei eigenständigen Sätzen sind typischerweise auch in asyndetischen Konstruktionen zu finden (vgl. 33b im nächsten Abschnitt).

Zusammenfassend können wir festhalten, dass die V-nach-C-Bewegung des finiten Verbs in V2-Nebensätzen genauso wie in V2-Hauptsätzen den Kontextindex $<$ Epist $>$ lizenziert. Da $<$ Epist $>$ zu einer Kontexterweiterung führt, darf die durch den V2-Satz denotierte Proposition nicht Teil des CG und damit nicht präsupponiert (oder diskurs-linked) sein. Aus diesem Grund sind WV2 im Skopus von Präsuppositionsauslösern wie iterativen Adverbien, Fragesätzen und präsupponierenden Fokuspartikeln ausgeschlossen (zu präsuppositionsauslösenden Ausdrücken vgl. Beaver 2001: 11). V2-Nebensätze erfahren im Gegensatz zu VLNebensätzen eine informationsstrukturelle Aufwertung, da sie in allen Kontexten ausgeschlossen sind, in denen sie Teil des gemeinsamen Hintergrundwissens sind. Aus diesem Grund erscheint die Distanzierung von $\mathrm{S}$ von einer mit einem WV2 realisierten propositionalen Begründung merkwürdig (vgl. 31a). Bei WVL wie (31b) ist dies hingegen problemlos möglich (vgl. Keller 1993: 231).
a. Hans ist nach Hause gegangen, weil er hatte Kopfweh. \#Aber ich glaube nicht, dass das der wirkliche Grund war.
b. Hans ist nach Hause gegangen, weil er Kopfweh hatte. Aber ich glaube nicht, dass das der wirkliche Grund war.

VL-Nebensätze sind bezüglich ihres assertiven Potentials neutral, weshalb sie (von den in Abschnitt 4 diskutierten spezifischen Interpretationen von WV2 einmal abgesehen) immer einen V2-Nebensatz ersetzen können. Die V2-Stellung hat in Nebensätzen somit im Wesentlichen eine disambiguierende Funktion (vgl. Pasch 1997: 267).

10. Dies gilt auch für sprechaktbezogene Adverbiale oder assertive Partikeln, die in allen drei Verwendungen von WV2 und auch in prosodisch desintegrierten WVL zu finden sind (vgl. auch Reis 2006). 
Zusammen mit den in Abschnitt 3 erwähnten syntaktischen Unterschieden zwischen V2- und VL-Nebensätzen können wir nun die in Tabelle 1 zusammengefassten spezifischen Eigenschaften der V2-Nebensätze ableiten. Einige dieser Eigenschaften ergeben sich aus dem assertiven Potential, das sich alle V2-Nebensätze teilen, andere aus ihrer spezifischen syntaktischen Struktur. Gehen wir zuerst auf das assertive Potential ein. Da V2-Nebensätze aufgrund des Kontextindexes $<$ Epist $>$ nicht Teil des CGs sein dürfen, können sie nicht im Skopus der Negation und von präsupponierenden Fokuspartikeln stehen. Genauso wenig können sie als Teil des Hintergrunds oder als Echofragen verwendet werden. Bei Korrelaten liegt eine ähnliche Beschränkung vor. Als anaphorische (oder kataphorische) Elemente präsupponieren Korrelate ein Antezedens und sind deshalb ebenfalls inkompatibel mit einem V2-Nebensatz. WV2 können zudem nicht in Interrogativ- und Imperativsätze integriert werden, da sie eigenständige assertive Sprechakte sind. Bei RV2 wird das assertive Potential von der Matrixassertion absorbiert, weshalb sie keine Konstituenten in Interrogativ- und Imperativsätzen modifizieren können. KV2 können im Gegensatz dazu in Interrogativ- und Imperativsätze integriert werden, da hier das assertive Potential vom Matrixverb absorbiert wird.

Die in Abschnitt 3 schon diskutierten syntaktischen Eigenschaften der verschiedenen V2-Nebensätze erklären, warum Quantoren/VariablenBindung bei KV2 möglich ist und warum V2-Nebensätze nicht mit VLNebensätzen koordiniert werden können. Assertierende Fokuspartikeln unterliegen syntaktischen und semantisch-pragmatischen Beschränkungen. Im Gegensatz zu den präsupponierenden Fokuspartikeln können sie prinzipiell in V2-Nebensätze binden. Dies ist allerdings nur dann möglich, wenn der V2-Nebensatz syntaktisch oder informationsstrukturell im Skopus der Fokuspartikel steht. Deshalb können assertierende Fokuspartikeln keine Konstituenten in WV2, sehr wohl aber Konstituenten in KV2 und RV2 binden.

Eine Eigenschaft, die noch offen ist, ist die obligatorische Nachstellung von V2-Nebensätzen. Für WV2 und RV2 ist diese Beschränkung unproblematisch, da sie direkt aus der parataktischen Struktur in (20a) folgt. Bei KV2 ist dies nicht möglich, da sie als Adjunkte prinzipiell vorfeldfähig sein sollten. Truckenbrodt (2006a: 287) argumentiert, dass KV2 zum einen nicht in satzinitialer Position interpretiert werden können, zum anderen aber auch nicht korrekt rekonstruiert werden können, da sie nach seiner Analyse sowohl in der Komplementposition als auch in der Adjunktposition interpretiert werden müssen. Eine alternative Möglichkeit wäre, die Nachstellung von V2-Nebensätzen generell aus ihrem assertiven Potential abzuleiten. Dies würde voraussetzen, dass für nichttopikalische Nebensätze nur die Nachfeldposition optimal ist und nur 
topikalische Nebensätze vorangestellt werden können. Wir lassen diese Frage hier offen, da sie die Analyse von WV2 nicht weiter betrifft.

In Abschnitt 3 haben wir schon erwähnt, dass sich WV2 von KV2 und RV2 durch ihren hohen Grad an Desintegration unterscheiden. Im Gegensatz zu KV2 und RV2 korrespondiert bei WV2 die in Abschnitt 3 diskutierte syntaktische Desintegration mit einer entsprechenden prosodischen und semantischen Desintegration. Die Desintegration geht bei WV2 so weit, dass mit ihnen ein eigenständiger assertiver Sprechakt ausgeführt werden kann. Da das assertive Potential des WV2 in (32) nicht durch den Interrogativsatz absorbiert werden kann, muss der weil-Satz wie ein uneingebetteter V2-Satz interpretiert werden.

Kannst du mir 10 Euro leihen? Weil ich hab mein Geld vergessen.

Der bisher diskutierte V2-Effekt ermöglicht es uns, die in Abschnitt 2 besprochenen Beschränkungen von V2-Nebensätzen abzuleiten. Die in Abschnitt 4 diskutierten Lesarten von WV2 folgen allerdings nicht direkt aus der V2-Stellung. Während RV2 und KV2 vor allem eine disambiguierende Funktion haben, indem sie das assertive Potential des Nebensatzes hervorheben, liegt die Sache bei WV2 etwas anders. Letztere unterscheiden sich von WVL nicht nur durch ihr assertives Potential, sondern auch durch zusätzliche Lesarten, die zumindest für integrierte WVL nicht möglich sind. Damit kommen wir zum zweiten Punkt, der Bedeutung der parataktischen Struktur von WV2.

\section{Desintegration und Interpretation}

In Abschnitt 5 haben wir gesehen, dass V2-Stellung in weil-Sätzen mit einem assertiven Potential verbunden ist. Außerdem führt die V2-Stellung zusammen mit der parataktischen Struktur zu einer starken syntaktischen Desintegration des Adverbialsatzes. Im Gegensatz dazu werden integrierte WVL adjungiert, so dass sie syntaktisch immer in den Matrixsatz integriert werden (vgl. 20). In diesem Abschnitt werden wir dafür argumentieren, dass die syntaktische Desintegration von WV2 auch eine semantisch-pragmatische Desintegration auslöst und so Basis für die in Abschnitt 4 beschriebenen Lesarten ist.

Die semantische Flexibilität von WV2 korrespondiert mit ihrer starken syntaktischen und semantisch-pragmatischen Desintegration (vgl. auch Scheutz 2001). Weniger stark verknüpfte Strukturen scheinen mehr interpretativen Spielraum zuzulassen als stärker verknüpfte Strukturen. Ein ähnlicher Unterschied findet sich in (33). Die koordinative Konstruktion in (33a) kann zu einer temporalen und kausalen Konjunktions- 
verstärkung (conjunction buttressing) führen, die Levinson (2000) als I-Implikatur analysiert: Der erste Satz gibt den Grund für den im zweiten Satz beschriebenen Sachverhalt an, d.h. es gab einen Unfall und dann/als Folge dessen ging der Airbag auf (vgl. auch Posner 1979). Im Gegensatz dazu kann die asyndetische Konstruktion in (33b) die entgegengesetze Implikatur auslösen. Der zweite Satz gibt hier den Grund für den im ersten Satz beschriebenen Sachverhalt an: Es gab einen Unfall, und der Grund dafür war ein aufgehender Airbag.

a. Es hat einen Unfall gegeben und der Airbag ist aufgegangen.

b. Es hat einen Unfall gegeben. Der Airbag ist aufgegangen.

Blakemore \& Carston (2004) und Carston (2002) argumentieren, dass die Bedeutungsunterschiede zwischen (33a) und (33b) aus den unterschiedlichen Verknüpfungen der beiden Sätze folgen. Etwas vereinfacht können wir ihren relevanztheoretischen Ansatz wie folgt zusammen fassen: Die beiden mit und verknüpften (integrierten) Sätze in (33a) bilden eine (pragmatische) Verarbeitungseinheit, wohingegen die beiden unverbundenen (desintegrierten) Sätze in (33b) zwei Verarbeitungseinheiten bilden (zu pragmatic processing units vgl. Carston 2002: Kap. 3). Daher sind in (33a) Inferenzen blockiert, die in (33b) möglich sind und umgekehrt. Die kausale Konjunktionsverstärkung setzt (abgesehen von einem unterstützenden Kontext) voraus, dass beide Sätze in einem Schritt verarbeitet werden. Im Gegensatz dazu setzt die umgekehrte kausale Implikatur in (33b) voraus, dass zunächst zwei getrennte Verarbeitungseinheiten generiert werden, die dann erst in einem zweiten Schritt aus Gründen der Textkohärenz aufeinander bezogen werden.

Interessanterweise entspricht die Interpretation in (33b) der salienten Interpretation des WV2 in (34b) als epistemische Einstellungsbegründung, derzufolge von einem aufgegangenen Airbag auf einen zeitlich vorher stattgefundenen Unfall geschlossen wird. Der integrierte WVL in (34a) wird im Gegensatz dazu als propositionale Begründung interpretiert: Der aufgegangene Airbag ist Ursache für den Unfall.

(34) a. Es hat einen Unfall gegeben, weil der Airbag aufgegangen ist.

b. Es hat einen Unfall gegeben, weil der Airbag ist aufgegangen.

Der interpretative Unterschied zwischen WV2 und integrierten WVL in (34) entspricht also in gewisser Hinsicht dem Unterschied zwischen der asyndetischen und der koordinativen Struktur in (33). Während WV2 eigenständige Assertionen und damit auch eigenständige Verarbeitungs- 
einheiten bilden, werden integrierte WVL zusammen mit ihrem Bezugssatz als eine komplexe Einheit verarbeitet. Allerdings bilden WV2 mit dem modifizierten Bezugssatz syntaktisch eine parataktische Struktur, die auf den ersten Blick große Ähnlichkeiten mit der und-Koordination in (33a) aufweist. Es gibt jedoch auch entscheidende Unterschiede zwischen den beiden koordinativen Konstruktionen (vgl. auch Breindl 2009). Im Gegensatz zu und können mit weil nur zwei selbstständige Matrixsätze koordiniert werden. Anders als und ist weil semantisch ein asymmetrischer Koordinator. Deshalb erlaubt weil, wie in (35) illustriert, keine prosodische Tilgung in einem der beiden Sätze (gapping und right node raising).
a. Er ist gestern die Treppe hinuntergefallen und er hat sich das Bein gebrochen.
b. *Er ist sicher die Treppe hinuntergefallen, weil er hat sich das Bein gebrochen.
c. Peter ist heute die Treppe runtergefallen und Maria ist ge- stern die Leiter runtergefallen.
d. *Peter ist heute die Treppe runtergefallen, weil Maria ist gestern die Leiter runtergefallen.

Zwei weitere Unterschiede bestehen darin, dass WV2 als eigenständige Assertionen mit ihrem Bezugssatz keine Verarbeitungseinheit bilden und dass für WV2 mit dem integrierten WVL in (34a) eine konkurrierende syntaktische Konstruktion vorliegt, die wesentlich stärker in den Matrixsatz integriert ist. Dies bedeutet, dass die Interpretation eines Satzes immer auch in Relation zu anderen vergleichbaren Konstruktionen berechnet werden muss. Abbildung 3 fasst die wesentlichen Unterschiede und Gemeinsamkeiten der und-Koordination und der WV2 zusammen.
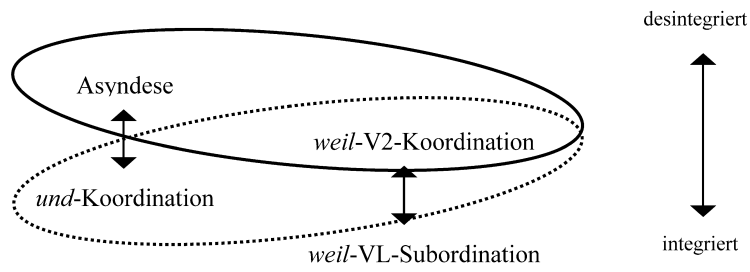

Abbildung 3: Einbettungsgrad und Interpretation

In stark desintegrierten Strukturen werden die beiden Sätze getrennt verarbeitet, in integrierten Strukturen dagegen zusammen. WV2 bilden demnach eine eigene Verarbeitungseinheit, die dann in einem weiteren Schritt vermittelt durch den kausalen Konnektor weil auf den Matrixsatz 
bezogen wird. Integrierte WVL werden im Gegensatz dazu immer zusammen mit ihrem Bezugssatz verarbeitet.

Wie werden die Argumente von WV2 und integrierten WVL nun konkret lizenziert? Das interne semantische Argument , $\mathrm{q}^{6}$ des zweistelligen kausalen Konnektors CAUSE(p,q) wird durch den Bezugssatz und das externe semantische Argument , $\mathrm{p}^{6}$ durch den weil-Satz lizenziert. Da integrierte WVL VP-Adjunkte sind (vgl. Struktur (20b)), kann (und muss) das interne Argument (i.e. q) in der Adjunktionsstruktur kanonischstrukturell lizenziert werden. Dies bedeutet, dass integrierte WVL als VP-Adjunkte nur die Proposition des Matrixsatzes modifizieren können und damit auf eine Interpretation als propositionale Begründungen festgelegt sind. Epistemische und sprechaktbezogene Begründungen sind somit ausgeschlossen, da für beide Interpretationen eine nichtkanonische Lizenzierung wie in WV2 nötig ist. Im Gegensatz zu integrierten WVL sind WV2 aufgrund ihrer parataktischen Struktur grundsätzlich ambig, da hier eine strukturelle Lizenzierung des internen Arguments nicht möglich ist. Aus diesem Grund muss dieses nichtkanonisch lizenziert werden. Eine nichtkanonische Lizenzierung eines internen propositionalen Arguments findet sich auch in KV2, RV2 und integrierten Parenthesen und scheint eine grundlegende Eigenschaft von eingebetteten V2-Sätzen zu sein (vgl. auch Reis 1995, 1997, Frank 2000, Gärtner 2001 und Steinbach 2007). Da WV2 parataktische Strukturen sind, werden sie zu einem Derivationszeitpunkt mit dem Bezugssatz verbunden, zu dem die $\mathrm{CP}$ des Bezugssatzes bereits abgeschlossen ist. Dadurch kann der Kausalsatz auch die Illokutionsebene modifizieren. Für die Sättigung der internen Argumentvariable stehen drei verschiedene Propositionen zur Verfügung (vgl. auch Pittner 1999: 339, Haegeman 2003 und Scheffler 2008: 78). Betrachten wir dazu die Definition einer Assertion bei Bach \& Harnish (1979: $42)$ in (36).

In uttering $e, \mathrm{~S}$ asserts that $q$ if $\mathrm{S}$ expresses:

(i) the belief that $q$, and

(ii) the intention that $\mathrm{A}$ believes that $q$.

Das im zweiten Konjunkt nicht lizenzierte semantische interne Argument des zweistelligen Konnektors (i.e. $\mathrm{x}$ in $\lambda \mathrm{x} \operatorname{CAUSE}(\mathrm{p}, \mathrm{x})$ ) kann nun durch unterschiedliche Propositionen lizenziert werden. Wird das interne Argument durch die Matrixproposition , $\mathrm{q}^{6}$ gesättigt, erhalten wir eine propositionale Begründung (vgl. 37a). Wird das interne Argument dagegen durch die epistemische Einstellung in (36(i)) gesättigt, ergibt sich eine epistemische Einstellungsbegründung wie in (37b). In der sprechaktbezo- 
genen Begründung wird, $\mathrm{x}^{6}$ wiederum wie in $(37 \mathrm{c})$ durch die gesamte Äußerung lizenziert. ${ }^{11}$

$$
\begin{array}{lll}
\text { a. } \quad \mathrm{x}=\mathrm{q} & \Rightarrow \operatorname{CAUSE}(\mathrm{p}, \mathrm{q}) & \begin{array}{l}
\text { propositionale } \\
\text { Begründung } \\
\text { epistemische }
\end{array} \\
\mathrm{b.} \quad \mathrm{x}=\mathrm{S} \text { glaubt dass } \mathrm{q} & \Rightarrow \operatorname{CAUSE}\left(\mathrm{p},\left(\mathrm{S}_{1} \text { glaubt } \mathrm{q}\right)\right) & \begin{array}{l}
\text { Begründung } \\
\text { sprechaktbezog. } \\
\text { Begründung }
\end{array} \\
\mathrm{c.} \quad \mathrm{x}=\mathrm{S} \text { äußert } \mathrm{e} & \Rightarrow \operatorname{CAUSE}\left(\mathrm{p},\left(\mathrm{S}_{1} \text { äußert }\right)\right.
\end{array}
$$

Zusammenfassend können wir festhalten, dass weil grundsätzlich eine zweistellige kausale Relation ausdrückt. Die Ambiguität des Konnektors entsteht durch unterschiedliche Belegungen der internen Argumentvariablen. In integrierten VL-Sätzen kann diese nur strukturell durch die Proposition des Matrixsatzes lizenziert werden. In desintegrierten V2Sätzen kann die Argumentposition hingegen durch unterschiedliche Propositionen lizenziert werden. ${ }^{12}$ Ein WV2 kann verschiedene Ebenen der vorangehenden Äußerung modifizieren. Unsere Analyse von WV2 und WVL basiert damit auf einer einheitlichen semantischen Grundrelation für alle drei Lesarten.

WV2 sind demnach potentiell ambig, da aus ihrem syntaktischen Status nicht hervorgeht, welches Argument sie modifizieren. Semantisch öffnet der WV2 eine unterspezifizierte kausale Relation $\lambda x \operatorname{CAUSE}(\mathrm{p}, \mathrm{x})$. Auf der Basis von pragmatischen Prinzipien wie dem Relevanzprinzip wird dann in einem zweiten Schritt bestimmt, welche der drei möglichen Propositionen für $\mathrm{x}$ eingesetzt wird (vgl. Haegeman 1991). Welche Faktoren bestimmen nun die Festlegung auf eine der drei möglichen Argumentvariablen?

In vielen Fällen wird bei WV2 eine der drei möglichen Lesarten durch den disambiguierenden Kontext festgelegt. Dies trifft vor allem auf sprechaktbezogene WV2 zu, da sie immer einen Adressatenbezug haben. Modifiziert ein WV2 einen Interrogativ- oder Imperativsatz, dann ist nur die sprechaktbezogene Begründung möglich. Disambiguiert der Kontext nicht ausreichend, kann die Verwendung von WV2 einen pragmatischen

11. Dieselben Interpretationen sind auch bei prosodisch desintegrierten WVL möglich (vgl. Abschnitt 4).

12. Eine mögliche Interpretation von WV2 ist die für WVL typische propositionale Begründung. In dieser Hinsicht unterscheiden sich WV2 nicht von asyndetischen Konstruktionen, die ebenfalls dieselbe Interpretation haben können wie die entsprechenden koordinativen Konstruktionen:

(i) a. Marlene fuhr nach Spanien und sie kaufte sich viele T-Shirts.

b. Marlene fuhr nach Spanien. Sie kaufte sich viele T-Shirts.

c. Marlene will nach Spanien, weil dort scheint immer die Sonne.

d. Marlene will nach Spanien, weil dort immer die Sonne scheint. 
Blocking-Effekt auslösen. Der Gebrauch eines ambigen WV2 löst die Implikatur aus, dass $\mathrm{S}$ eine epistemische Lesart intendiert, da die propositionale Lesart durch die nichtambige integrierte WVL-Variante eindeutig markiert wird. Blühdorn (2008) spricht in diesem Kontext von einer pragmatischen Spezialisierung von WV2 aufgrund der Griceschen Maximen. Dadurch besteht in ambigen Kontexten eine Präferenz für die epistemische Interpretation von WV2. Dieser pragmatische Blocking-Effekt ist zwar schwach, aber, wie wir im nächsten Abschnitt zeigen werden, durchaus nachweisbar.

In welchem Verhältnis stehen nun V2-Stellung, Assertivität und die verschiedenen Lesarten zueinander? Wie in Abschnitt 5 gezeigt, ist V2Stellung in weil-Sätzen durch die assertive Semantik des Satzes lizenziert, was daran deutlich wird, dass V2-Stellung in präsupponierten Kausalsätzen blockiert ist. Die Verwendung eines WV2 entspricht demnach dem Wunsch von S, den CG zu erweitern und dieses Kontextupdate als solches zu markieren. Mit der Bewegung von V-nach-C ist eine syntaktische und informationsstrukturelle Aufwertung des Satzes verbunden, der als Konjunkt einer parataktischen Struktur ein eigenes illokutionäres Potential hat. Als Folge der syntaktischen Desintegration können WV2 Propositionen modifizieren, die für integrierte WVL nicht zugängig sind. Die Tatsache, dass WV2 gut geeignet sind für den Ausdruck von epistemischen und sprechaktbezogenen Begründungen, ist folglich ein Nebenprodukt der durch $<$ Epist $>$ lizenzierten V-nach C-Bewegung. Dass V2-Stellung durch das assertive Potential und nicht durch die epistemische oder sprechaktbezogene Lesart des Satzes ausgelöst wird, zeigt folgendes Beispiel. In Beispiel (38) handelt es sich um eine sprechaktbezogene Begründung, dennoch ist VL-Stellung präferiert, da der Kausalsatz einen gegebenen Sachverhalt (der bereits Teil des CG ist) ausdrückt.

(38) [Sabine und Peter unterhalten sich beim Abendessen über Vornamen. Peter:] Claudia hat ihren Sohn übrigens Paul genannt, (\) weil wir (\#reden) ja gerade die ganze Zeit über Vornamen (reden).

Wie bereits in Abschnitt 4 erwähnt, können auch WVL sprechaktbezogen oder epistemisch verwendet werden, wenn sie intonatorisch desintegriert sind. Bei WV2 handelt es sich allerdings um optimal desintegrierte Strukturen, da sie im Vergleich zu WVL auch syntaktisch desintegriert sind. WVL werden demnach vor allem dann zum Ausdruck epistemischer oder sprechaktbezogener Begründungen verwendet, wenn der Kausalsatz einen gegebenen Sachverhalt ausdrückt wie in Beispiel (38) oder 
wenn der Sprachgebrauch den normativen Regeln der Schriftsprache unterliegt.

Zusammenfassend können wir festhalten, dass integrierte WVL einerseits nur eine der drei möglichen Lesarten von WV2 erhalten können. Somit bildet die Interpretation von integrierten WVL eine Untermenge der Interpretationen von WV2. Andererseits sind WV2 immer assertiv. Damit bilden sie in dieser Hinsicht eine Untermenge der diesbezüglich neutralen WVL. Assertive weil-Sätze mit einer propositionalen Lesart bilden demnach die Schnittmenge zwischen WV2 und WVL.

\section{WV2 empirisch}

In einer ersten kleinen Pilotstudie zu WV2 haben wir getestet, ob sich die in den Abschnitten 5 und 6 diskutierten Unterschiede empirisch nachweisen lassen. Aus unserer Analyse von WV2 ergibt sich zum einen die Annahme, dass WV2 nicht präsupponiert (oder diskurs-linked) sein dürfen. Zum anderen sagen wir Unterschiede zwischen WVL und WV2 in Bezug auf die Zugänglichkeit der verschiedenen Lesarten voraus. Für die Untersuchung der ersten Annahme wurden assertive WV2 mit WV2, die im Skopus eines Präsuppositionsauslösers stehen, kontrastiert. So ist der nachgestellte V2-Satz in Beispiel (39a) in einen Interrogativsatz integriert und in (39b) hat die Matrixnegation Skopus über den weilSatz. In dem Testsatz (39c) dagegen ist eine assertive Lesart des WV2 möglich.

(39) a. \#Bist du so nervös, weil deine Schwiegermutter kommt zu Besuch?

b. *Ich bin dir nicht böse, weil du hast meinen Geburtstag vergessen.

c. Lass uns lieber heute etwas unternehmen, weil morgen bin ich schon verabredet.

Die Versuchsteilnehmer, 148 Studierende der Johannes Gutenberg-Universität Mainz, wurden aufgefordert, den insgesamt 6 Sätzen in Relation zu einem vorgegebenen, grammatisch einwandfreien Beispielsatz proportional zu dem von ihnen empfundenen Akzeptabilitätsgrad Zahlen zuzuordnen. Diese Erhebungsmethode ist eine leicht modifizierte Variante von Magnitude Estimation (vgl. Sorace \& Keller 2005), der ein gradueller Grammatikalitätsbegriff zugrunde liegt. Anders als in klassischen Erhebungen mittels Magnitude Estimation haben wir es den Versuchsteilnehmern jedoch nicht überlassen, dem Bezugssatz selbst einen Wert zuzuordnen. Stattdessen haben wir das obere Ende der Bewertungsskala vorgegeben, indem wir dem einwandfreien Vergleichssatz den Wert 10 
zugeordnet haben (vgl. Featherston 2007: 296). Diese Änderung mussten wir vornehmen, um auszuschließen, dass für den perfekten Vergleichssatz ein zu geringer Wert gewählt würde, was eine starke Einschränkung der möglichen Abstufungen in der Bewertung von als weniger gut empfundenen Sätzen zur Folge hätte. ${ }^{13}$

Die Ergebnisse des ersten Teils der Erhebung korrelieren mit der von uns getroffenen Voraussage, da sich ein signifikanter Akzeptabilitätsunterschied zwischen den drei untersuchten Faktoren Negation, Integration in einen Interrogativsatz und assertives Potential feststellen lässt. ${ }^{14}$ In Tabelle 3 sind für alle drei Beispieltypen aus (39) die Ergebnisse angegeben. Wie vorausgesagt wurden WV2, die in einen Interrogativsatz integriert sind oder im Skopus der Matrixnegation stehen, als deutlich schlechter empfunden als assertive WV2.

Tabelle 3: Akzeptanz von präsupponierten und assertiven WV2

\begin{tabular}{|l|c|c|c|}
\hline & Interrogativsatz & Negation & Assertion \\
\hline Mittelwert & 2,01 & 2,75 & 7 \\
\hline Median & 2 & 3 & 8 \\
\hline
\end{tabular}

Für die Untersuchung der zweiten Behauptung haben wir ambige Sätze konstruiert, die sowohl epistemisch als auch propositional interpretiert werden können. In zwei verschiedenen Fragebögen A und B wurden die Versuchsteilnehmer aufgefordert, die Bedeutung der jeweiligen Sätze mit eigenen Worten zu paraphrasieren, wobei jeder der vier getesteten weilSätze für die Hälfte der Probanden als VL- und für die andere Hälfte als V2-Variante vorlag.

(40) a. Es hat einen Unfall gegeben, weil der Airbag ist aufgegangen.

b. Es hat einen Unfall gegeben, weil der Airbag aufgegangen ist.

Die Überprüfung der Zugänglichkeit der epistemischen und der propositionalen Lesarten von WV2 und WVL hat zu folgenden Ergebnissen geführt: Nur etwa ein Drittel der Versuchsteilnehmer haben sich bei dem

13. Zudem muss berücksichtigt werden, dass die Beurteilung der Daten durch präskriptivnormative Faktoren beeinflusst werden kann, zumal die Studie schriftlich durchgeführt wurde, es sich bei WV2 aber um ein Phänomen der Mündlichkeit handelt. Durch eine weit gefasste Akzeptabilitätsskala, die Raum für graduelle Abstufungen der Urteile zulässt, sowie durch eine relationale Bewertung kann allerdings eine auf präskriptiv definierter „Korrektheit“ basierende Ablehnung sämtlicher WV2 verhindert werden.

14. Eine vergleichbare Studie mit 70 Schülerinnen und Schülern zwischen 11 und 17 Jahren hat zu ähnlichen Ergebnissen geführt. 
WVL in (40b) für eine epistemische Lesart entschieden, wohingegen knapp 72 Prozent der Versuchsteilnehmer den entsprechenden WV2 (40a) als epistemische Einstellungsbegründung interpretiert haben. Die Gesamtergebnisse der Untersuchung von insgesamt vier ambigen Sätzen sind in Abbildung 4 dargestellt.

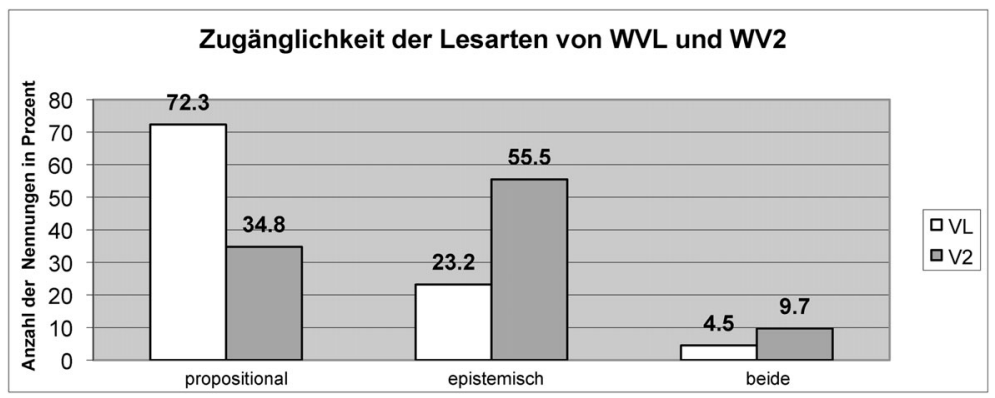

Abbildung 4: Zugänglichkeit der Lesarten von WVL und WV2

Die Ergebnisse zeigen, dass die in den Abschnitten 4 und 6 getroffenen Voraussagen über die Zugänglichkeit der unterschiedlichen Lesarten von weil-Sätzen zutreffend sind. So wurden WVL präferiert propositional interpretiert. Allerdings wählten die Probanden in immerhin 23,2 Prozent der Fälle eine Lesart des WVL als epistemische Einstellungsbegründung, auch wenn eine propositionale Interpretation des Satzes möglich war. Wie in Abschnitt 4 gezeigt, können aber nur intonatorisch desintegrierte WVL die epistemische Lesart zulassen. Aufgrund der schriftlichen Durchführung der Untersuchung und dem Fehlen eindeutiger Indikatoren für Integration (wie z. B. Voranstellung oder Korrelate) können wir jedoch keine Aussage darüber machen, ob den Interpretationen eine integrierte oder desintegrierte Struktur zugrunde lag. Um die Frage definitiv zu klären, ist die Durchführung weiterer Experimente mit gesprochenen Stimuli notwendig.

Bei WV2 kehrt sich das Verhältnis um: Über die Hälfte (55,5 Prozent) erhielten eine epistemische Lesart und nur 34,8 Prozent der Sätze wurden auf die propositionale Ebene bezogen. Dieses Ergebnis spricht für einen wie in Abschnitt 6 beschriebenen pragmatischen Blocking-Effekt. Auffällig ist außerdem, dass in 9,7 Prozent aller V2-Nebensätze beide Lesarten erkannt wurden, bei den entsprechenden WVL aber nur in 4,5 Prozent der Fälle. Das zeugt von einer größeren semantischen und pragmatischen Flexibilität der V2-Nebensätze im Vergleich zu den kanonischen WVL. Unterschiede zwischen den Ergebnissen der vier ambigen Testsätze zeigen außerdem, dass neben der Position des Finitums Kontextbedingungen die epistemische beziehungsweise die propositionale Lesart begünstigen können. 


\section{Zusammenfassung}

In diesem Aufsatz haben wir gezeigt, dass sich WV2 nicht nur formal, sondern auch funktional von den entsprechenden integrierten WVL unterscheiden. Tabelle 4 gibt einen Überblick über die wesentlichen Ergebnisse. Da WV2 parataktische Strukturen sind, sind sie im Vergleich mit subordinierten WVL syntaktisch stärker desintegriert. Damit einher geht bei WV2 normalerweise auch eine prosodische Desintegration. Die V2Stellung lizenziert in WV2 das Kontextmerkmal <Epist>, so dass der eingebettete V2-Satz im Gegensatz zu seinem VL-Pendant ein assertives Potential erhält. Die desintegrierte Struktur von WV2 erzwingt zudem eine pragmatische Anreicherung bei der Interpretation des kausalen Bezugs, da das ungesättigte interne Argument des Konnektors nicht kanonisch lizenziert werden kann. Daher können WV2 drei unterschiedliche Lesarten erhalten, wohingegen für integrierte WVL nur eine propositionale Interpretation möglich ist. Zudem ist bei ambigen WV2 ein leichter Blocking-Effekt zu beobachten, der durch die Konkurrenz mit den eindeutig integrierten WVL entsteht.

Tabelle 4: Die Syntax, Semantik und Pragmatik von weil-VL- und weil-V2-Sätzen

\begin{tabular}{|l|l|l|}
\hline \multirow{2}{*}{ Syntax } & \multicolumn{1}{|c|}{ integrierte WVL } & \multicolumn{1}{c|}{ WV2 } \\
\hline Semantik/Pragmatik & $\begin{array}{l}\text { Subordination (VL) } \\
\text { integriert }\end{array}$ & $\begin{array}{l}\text { Koordination (V2) } \\
\text { desintegriert }\end{array}$ \\
\cline { 2 - 3 } & + keine Ansertiv & + assertiv \\
\cline { 2 - 3 } & propositional & $\begin{array}{l}\text { Anreicherung } \\
\text { propositional, epistemisch, } \\
\text { sprechaktbezogen }\end{array}$ \\
\hline Flexibilität & syntaktisch/semantisch & semantisch/pragmatisch \\
\hline
\end{tabular}

WV2 sind aufgrund ihrer Desintegration im Vergleich zu integrierten WVL in Bezug auf die Lesarten semantisch und pragmatisch flexibler. Umgekehrt verfügen integrierte WVL über eine größere syntaktische und zudem auch über eine gewisse semantische Flexibilität, da sie aufgrund der VL-Stellung nicht notwendigerweise assertiv sind. Diese Ergebnisse wurden im Wesentlichen durch eine kleine empirische Studie belegt.

Da unsere Analyse WV2 generell im Kontext von anderen eingebetteten V2-Sätzen untersucht, kann sie die Unterschiede zwischen WV2 und WVL ohne konstruktionsspezifische Annahmen erklären. Stattdessen 
werden die spezifischen Eigenschaften von WV2 systematisch aus der Interaktion von Syntax, Semantik und Pragmatik abgeleitet.

Eingereicht: 8. September 2008

Überarbeitete Fassung eingereicht:

9. September 2009
Seminar für Deutsche Philologie

Georg-August-Universität Göttingen markus.steinbach@phil.uni-goettingen.de mailin.antomo@phil.uni-goettingen.de

\section{Literatur}

Altmann, Hans (1993). Satzmodus. In Syntax. Ein internationales Handbuch zeitgenössischer Forschung, Joachim Jacobs et al. (eds.), 1006-1029. Berlin: Mouton de Gruyter.

Antomo, Mailin (2007). Eingebettete Verbzweitsätze im Deutschen - „Weil das ist ein Nebensatz". Staatsexamensarbeit, Gutenberg-Universität Mainz.

Bach, Kent \& Robert M. Harnish (1979). Linguistic Communication and Speech Acts. Cambridge, Mass: MIT Press.

Ballweg, Joachim (2004). Weil - Ursachen, Gründe, Motive. In Brücken schlagen - Grundlagen der Konnektorensemantik, Hardarik Blühdorn et al. (eds.), 325-332. Berlin: Walter de Gruyter.

Beaver, David (2001). Presupposition and Assertion in Dynamic Semantics. Stanford: CSLI Publications.

Blakemore, Diane \& Robyn Carston (2004). The pragmatics of sentential coordination with "and". Lingua 115: 569-589.

Blühdorn, Hardarik (2006). Kausale Satzverknüpfungen im Deutschen. Pandaemonium Germanicum. Revista de Estudos Germanísticos 10: 253-282.

Blühdorn, Hardarik (2008). Epistemische Lesarten von Satzkonnektoren - Wie sie zustande kommen und wie man sie erkennt. In Semantik und Pragmatik - Schnittstellen, Inge Pohl (ed.), 217-251. Frankfurt, Main: Lang.

Brandt, Margareta, Marga Reis, Inger Rosengren \& Ilse Zimmermann (1992). Satztyp, Satzmodus und Illokution. In Satz und Illokution. Band 1, Inger Rosengren (ed.), 1-90. Tübingen: Niemeyer.

Breindl, Eva (2009). Fehler mit System und Fehler im System. Topologische Varianten bei Konnektoren. In Deutsche Grammatik - Regeln, Normen, Sprachgebrauch, Marek Konopka \& Bruno Strecker (eds.), 274-306. Berlin: Walter de Gruyter.

Breul, Carsten (1997). Grammatik und Bedeutung der kausalen Satzverbände. "Because", "as", "since" und "for" im schriftsprachlichen Englisch. Tübingen: Niemeyer.

Buscha, Joachim (1989). Lexikon deutscher Konjunktionen. Leipzig: VEB.

Carston, Robyn (2002). Thoughts and Utterances. The Pragmatics of Explicit Communication. Oxford: Blackwell.

Featherston, Sam (2007). Data in generative grammar: The stick and the carrot. Theoretical Linguistics 33: 269-318.

Frank, Nicola (2000). Probleme lexikalischer Selektion und abhängige Verbzweitsätze. Linguistische Berichte 184: 469-483.

Gärtner, Hans-Martin (2001). Are there V2 relative clauses in German? Journal of Comparative Germanic Linguistics 3: 97-141.

Gärtner, Hans-Martin (2002). On the force of V2 declaratives. Theoretical Linguistics 28: $33-42$.

Gärtner, Hans-Martin \& Markus Steinbach (2003). What do reduced pronominals reveal about the syntax of Dutch and German? Part 2: Fronting. Linguistische Berichte 196: $459-490$.

Gaumann, Ulrike (1983). Weil die machen jetzt bald zu. Angabe- und Junktivsatz in der deutschen Gegenwartssprache. Göppingen: Kümmerle. 
Graf, Claudia (2007). Echo und Zitat. In Zitat und Bedeutung, Elke Brendel, Jörg Meibauer \& Markus Steinbach (eds.), 89-109. Hamburg: Buske.

Günthner, Susanne (1993). „... weil - man kann es ja wissenschaftlich untersuchen“ Diskurspragmatische Aspekte der Wortstellung in WEIL - Sätzen. Linguistische Berichte 143: 37-59.

Günthner, Susanne (1999). Entwickelt sich der Konzessivkonnektor obwohl zum Diskursmarker? Grammatikalisierungstendenzen im gesprochenen Deutsch. Linguistische Berichte 180: 409-446.

Haegeman, Liliane (1991). Parenthetical adverbials: The radical orphanage approach. In Aspects of Modern English Linguistics, S. Chiba et al. (eds.), 232-254. Tokio: Kaitakusha.

Haegeman, Liliane (2003). Conditional clauses: External and internal syntax. Mind \& Language 18: 317-339.

Haegeman, Liliane (2006). Conditionals, factives and the left periphery. Lingua 116: $1651-1669$.

Heycock, Caroline (2005). Embedded root phenomena. In The Blackwell Companion to Syntax, Martin Everaert \& Henk van Riemsdijk (eds), 174-209. Oxford: Blackwell.

Höhle, Tilmann (1986). Der Begriff ,Mittelfeld‘: Anmerkungen über die Theorie der topologischen Felder. In Textlinguistik contra Stilistik, Walter E. Weiss et al. (eds.), 329-340. Tübingen: Niemeyer.

Holler, Anke (2008). German dependent clauses from a constraint-based perspective. In 'Subordination' vs. 'Coordination' in Sentence and Text: A Cross-Linguistic Perspective, Cathrine Fabricius-Hansen (ed.), 187-216. Amsterdam: John Benjamins.

Hooper, Joan \& Sandra Thompson (1973). On the applicability of root transformations. Linguistic Inquiry 4: 465-497.

Iwata, Seizi (2003). Echo questions are interrogatives? Another version of a metarepresentational analysis. Linguistics and Philosophy 26: 185-254.

Keller, Rudi (1993). Das epistemische weil - Bedeutungswandel einer Konjunktion. In Sprachgeschichte und Sprachkritik. Festschrift für Peter von Polenz, Hans Jürgen Heringer \& Georg Stötzel (eds.), 219-247. Berlin: Mouton de Gruyter.

Küper, Christoph (1991). Geht die Nebensatzstellung im Deutschen verloren? Zur pragmatischen Funktion der Wortstellung in Haupt- und Nebensätzen. Deutsche Sprache 19: $133-158$.

Küper, Christoph (1993). Pragmatische Motiviertheit in der Syntax. Haupt- und Nebensätze im Deutschen. In Von der Sprache zur Literatur. Motiviertheit im sprachlichen und poetischen Kode. Christoph Küper (ed.), 37-49. Tübingen: Stauffenburg.

Levinson, Stephen C. (2000). Presumptive Meanings: The Theory of Generalized Conversational Implicatures. Cambridge, Mass: MIT Press.

Lohnstein, Horst (2000). Satzmodus - kompositionell. Zur Parametrisierung der Modusphrase im Deutschen. Berlin: Akademie.

Lohnstein, Horst (2004). Variable und invariante Strukturmerkmale von Satzkonnektoren. In Brücken schlagen - Grundlagen der Konnektorensemantik, Hardarik Blühdorn et al. (eds.), 137-160. Berlin: Walter de Gruyter.

Meinunger, André (2004). Verb position, verbal mood and the anchoring (potential) of sentences. In The Syntax and Semantics of the Left Periphery, Horst Lohnstein \& Susanne Trissler (eds.), 313-342. Berlin: Mouton de Gruyter.

Pittner, Karin (1999). Adverbiale im Deutschen. Untersuchungen zu ihrer Stellung und Interpretation. Tübingen: Stauffenburg.

Pasch, Renate (1997). Weil mit Hauptsatz - Kuckucksei im denn-Nest. Deutsche Sprache 25: $252-271$.

Pasch, Renate, Ursula Brauße, Eva Breindl \& Ulrich Hermann Waßner (Hgg.) (2003). Handbuch der deutschen Konnektoren. Berlin: Mouton de Gruyter. 
Posner, Roland (1979). Bedeutung und Gebrauch der Satzverknüpfer in den natürlichen Sprachen. In Sprechakttheorie und Semantik, Günther Grewendorf (ed.), 345-385. Frankfurt, Main: Suhrkamp.

Reich, Ingo (2008). „Asymmetrische Koordination“ im Deutschen. Tübingen: Stauffenburg.

Reis, Marga (1992). Zur Grammatik und Pragmatik von Echo-w-Fragen. In Satz und Illokution. Band 1, Inger Rosengren (ed.), 213-262. Tübingen: Niemeyer.

Reis, Marga (1995). Extraction from verb-second clauses in German? In On Extraction and Extraposition in German, Uli Lutz \& Jürgen Pafel (eds.), 45-88. Amsterdam: John Benjamins.

Reis, Marga (1997). Zum syntaktischen Status unselbstständiger Verbzweit-Sätze. In Syntax im Fokus, Christa Dürscheid et al. (eds.), 112-144. Tübingen: Niemeyer.

Reis, Marga (2006). Is German V-to-C-movement really semantically motivated? Some empirical problems. Theoretical Linguistics 22: 369-380.

Scheffler, Tatjana (2008). Semantic operators in different dimensions. Unpublished PhD, University of Pennsylvania.

Scheutz, Hannes (1998). Weil-Sätze im gesprochenen Deutsch. In Beiträge zur Dialektologie des ostoberdeutschen Raumes, Claus Hutterer \& Gertrude Pauritsch (eds.), 85-112. Göppingen: Kümmerle.

Scheutz, Hannes (2001). The case of spoken weil in German. In Studies in Interactional Linguistics, Margret Seltling \& Elizabeth Couper-Kuhlen (eds.), 111-139. Amsterdam: John Benjamins.

Sorace, Antonella \& Frank Keller (2005). Gradience in linguistic data. Lingua 115: $1497-$ 1524.

Sweetser, Eve (1990). From Etymology to Pragmatics. Metaphorical and Cultural Aspects of Semantic Structure. Cambridge: Cambridge University Press.

Stalnaker, Robert C. (2002). Common ground. Linguistics and Philosophy 25, 701-721.

Steinbach, Markus (2007). Integrated parentheticals and assertional complements. In Parentheticals, Nicole Dehé \& Yordanka Kavalova (eds.), 53-87. Amsterdam: John Benjamins.

Thim-Mabrey, Christiane (1982). Zur Syntax der kausalen Konjunktionen weil, da und denn. Sprachwissenschaft 7: 197-219.

Truckenbrodt, Hubert (2006a). On the semantic motivation of syntactic verb movement to $\mathrm{C}$ in German. Theoretical Linguistics 22: 257-306.

Truckenbrodt, Hubert (2006b). Replies to the comments by Gärtner, Plunze and Zimmermann, Portner, Potts, Reis, and Zaefferer. Theoretical Linguistics 22: 387-410.

Uhmann, Susanne (1998). Verbstellungsvariation in weil- Sätzen: Lexikalische Differenzierung mit grammatischen Folgen. Zeitschrift für Sprachwissenschaft 17: 92-139.

Volodina, Anna (2009). Zur syntaktischen und prosodischen Markierung pragmatischer Phänomene im Bereich der Konnektorenforschung. In An der Grenze zwischen Grammatik und Pragmatik, Rita Brdar-Szabó (ed.), 151-159. Frankfurt, Main: Lang.

Wechsler, Stephen (1991). Verb second and illocutionary force. In Views on Phrase Structure, Katherine Leffel \& Denis Bouchard (eds.), 177-191. Dordrecht: Kluwer.

Wegener, Heide (1993). Weil - das hat schon seinen Grund. Zur Verbstellung in Kausalsätzen mit weil im gegenwärtigen Deutsch. Deutsche Sprache 21: 289-305.

Wegener, Heide (2000). Da, denn und weil - der Kampf der Konjunktionen. Zur Grammatikalisierung im kausalen Bereich. In Deutsche Grammatik in Theorie und Praxis, Rolf Thieroff (ed.), 69-81. Tübingen: Niemeyer. 